\title{
Evolution of the fingering pattern of an impacting drop
}

\author{
S. T. Thoroddsen \\ Department of Theoretical and Applied Mechanics, University of Illinois at Urbana-Champaign, Urbana, \\ Illinois 61801-2935
}

J. Sakakibara

Institute of Engineering Mechanics, University of Tsukuba, Tsukuba, Japan 305

(Received 25 August 1997; accepted 25 February 1998)

\begin{abstract}
The impact of a drop on a solid surface generates a rapidly expanding thin jet traveling along the surface. We study the evolution of the fingering pattern at the edge of this jet during the impact of a water drop on a glass plate. Multiple-flash photography shows that systematic changes in frontal shapes take place during the expansion. The initial fingers widen and split in two. This splitting is in many cases limited to the development of a double peak on each finger. The subsequent interaction of two such adjacent undulations often results in merging which produces three pronounced fingers. Despite the significant changes in the frontal shapes, the number of fundamental undulations remains approximately constant during the expansion. The progenitors of these azimuthal disturbances are observed right at first contact. Some heuristic arguments based on capillary waves are put forth to explain the splitting and merging. The main focus of this study is on impacts having Reynolds numbers of about 15000 , based on the drop diameter. The corresponding Weber numbers are about 1000. () 1998 American Institute of Physics.
\end{abstract}

[S1070-6631(98)02606-3]

\section{INTRODUCTION}

The impact of a drop on a solid surface is characterized by a very sudden transfer of momentum from the vertical to the horizontal direction. For large impact Reynolds numbers the horizontal fluid motion is initially confined to a thin jet traveling along the surface. This jet can travel even faster than the incoming drop. For low impact Reynolds numbers the expanding jet remains axisymmetric. However, as the impact Reynolds number becomes sufficiently large the expanding jet loses axisymmetry and undulations are observed both in the radial location of the contact line as well as the thickness of the expanding lamella. Here we study the shape and evolution of these frontal undulations. By using multiple flashes with an adjustable time delay, we are able to follow the exact evolution of the frontal shapes during the spreading. Figure 1 shows such a photograph of the lamellar expansion, using a quadruple-flash exposure.

Previous work on drop impacts comes from a variety of disparate sources, such as rain erosion, printing, spray cooling, coating, and cleaning. All this has recently been collected in a comprehensive review by Rein. ${ }^{1}$ The radial evolution of the fingering instability studied here has, however, not been investigated previously in any detail.

High Reynolds number impacts on dry surfaces were first studied by Worthington. ${ }^{2,3}$ He drew interesting pictures from direct visual observations of the impact of a mercury drop on a glass plate. He used an intricate mechanical contraption, using a marble, falling in tandem with the drop, to trigger a spark to freeze the drop shape. His drawings show clear azimuthal patterns, with thin ridges extending radially along the fluid film with fingers at the edge. Rein ${ }^{4}$ suggests that these ridges may be artifacts from the visualization method, i.e., from the spark light lasting too long. The spark was generated by pulling a charged wire out of a bath of mercury.

The work of Loehr and Lasek ${ }^{5}$ contains many interesting ideas, some of which have been pursued in Loehr's ${ }^{6}$ unpublished thesis. The number of fingers or azimuthal disturbances is there shown to remain constant during the spreading of the lamella, as described by Rein, ${ }^{4}$ without details of the experimental configuration. Loehr ${ }^{6}$ suggests that the number of fingers scales linearly with Reynolds number based on drop diameter. ${ }^{1}$ Marmanis and Thoroddsen ${ }^{7}$ showed subsequently that this number depends on surface tension in addition to the fluid viscosity and inertia. Allen ${ }^{8}$ has suggested that a Rayleigh-Taylor-type instability selects the number of fingers. This instability acts at the decelerating edge of the expanding jet and is modified by surface tension. Viscosity is not included in this analysis, contrary to the current experimental evidence.

The effects of surface roughness on the lamella have been studied by Stow and Hadfield. ${ }^{9}$ Photographs therein show dramatically that for a sufficiently rough surface the tip of the jet can leave the surface. Mundo, Sommerfeld, and Trope ${ }^{10}$ have studied the boundary between deposition and splashing of droplets impinging at various angles onto a rotating disk. Our video images taken from the side show no clear separation from the surface, in the cases studied here, neither for the anodized aluminum nor the glass surface.

Chandra and Avedisian ${ }^{11}$ have studied drop impacts at lower Reynolds numbers than studied here, producing an extraordinary set of photographs. Pasandideh-Fard et al. ${ }^{12}$ have similarily studied the dynamics of contact angles and the 


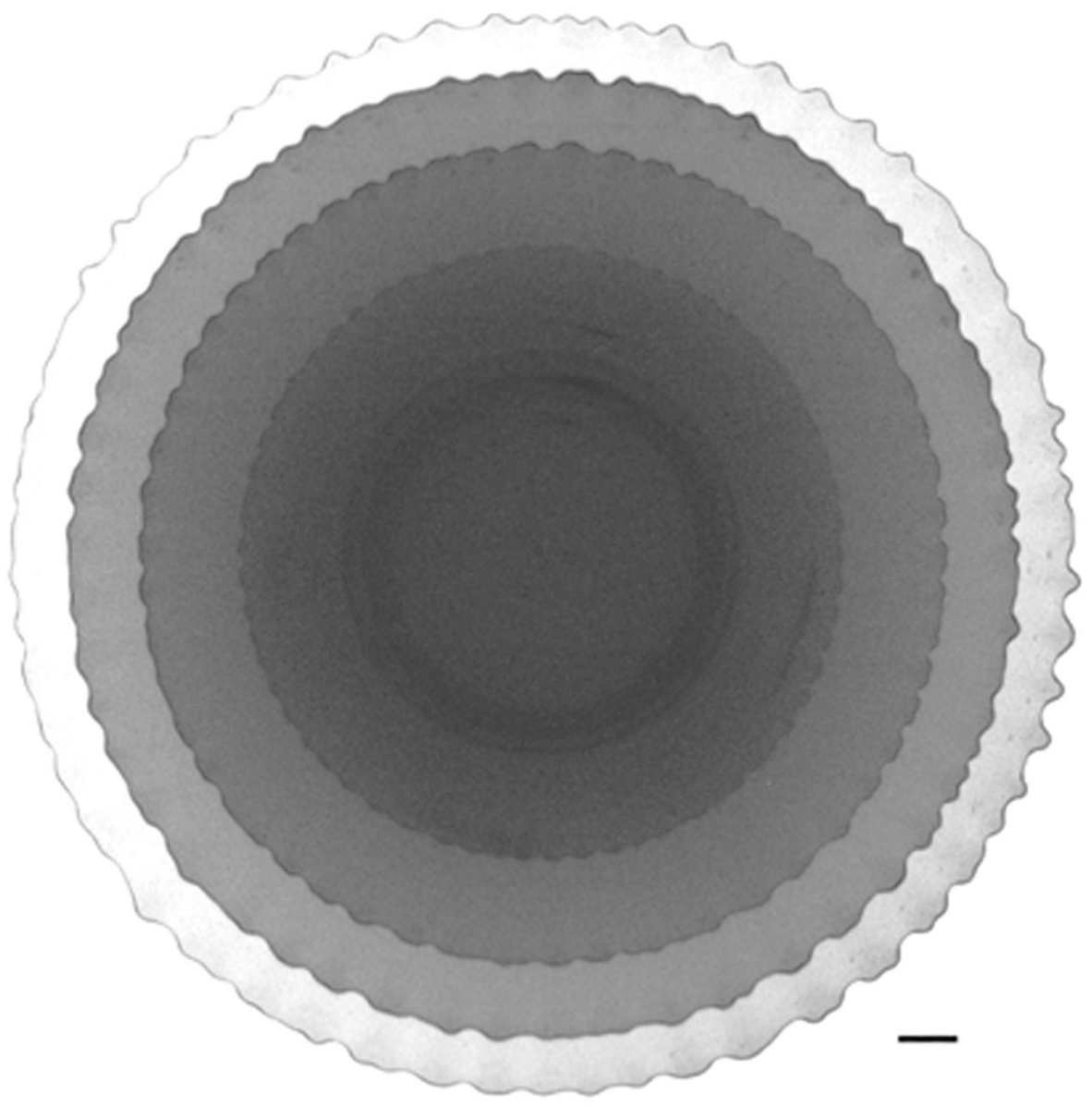

FIG. 1. A photograph (negative) showing the typical evolution of the frontal undulations during the spreading of the lamella. The four separate flashes illuminated the drop with time separation $\Delta \tau_{i}=0.6 \mathrm{~ms}$, with the first flash at $1.4 \mathrm{~ms}$ after the initial contact. Drop diameter $D=5.2 \mathrm{~mm}$ and release height $H=28.8 \mathrm{~cm}$. The scale bar is $1 \mathrm{~mm}$ long.

effects of surfactants. Owing to the lower Reynolds numbers the lamella remains axisymmetric in these studies.

A recent comprehensive study by Zhang and Basaran ${ }^{13}$ has investigated how the spreading and rebounding of a drop is affected by two different common surfactants. They find that the rebounding is more affected by the surfactants than the spreading phase. They point out that these effects are of considerable commercial interest, as the effectiveness of sprays, used, for example, in coating, cooling, or delivery of agricultural chemicals, might easily be improved by the addition of surfactants to the liquid.

Numerical simulations of drops impacting on solids were begun by Harlow and Shannon, ${ }^{14}$ who applied particlein-cell methods. More recent work focuses on problems with practical applications, ${ }^{15,16}$ such as the impact of a drop of molten tin. These simulations attack the simplified axisymmetric problem. The unknown boundary conditions at the rapidly moving contact line presents a particular challenge for simulations. Experimentally determined dynamic contact angles have in some cases been used for boundary conditions. ${ }^{12}$

The impact of a drop onto a layer of fluid has been extensively studied and will not be reviewed here. Rein's ${ }^{1}$ review is an excellent source for work in this area. The for- mation of liquid crowns is particularly interesting and has recently been related to the formation of a kinematic singularity. ${ }^{17}$

\section{THE EXPERIMENTS}

Figure 2 shows schematically the experimental setup, identifying the timing and optical systems. The setup is fashioned after the one presented by Chandra and Avedisian. ${ }^{11}$ Peck and Sigurdson ${ }^{18}$ have used a similar, but completely automated, system for the study of the vortex ring generated by a drop entering a deep fluid.

\section{A. Triggering mechanism}

The drop is released from the circular opening of a plastic gate valve. The inner diameter of the opening is $4.5 \mathrm{~mm}$, with a wall thickness of about $0.8 \mathrm{~mm}$. The flow rate through the valve is adjusted to be very slow, to allow the balance between gravity and surface tension to control the release, from the outer edge of the opening. Weight measurements were used to determine the drop size, giving a size of around $5 \mathrm{~mm}$, depending somewhat on the surface tension. The drop interrupts a laser beam sensed by a photodiode, which begins 


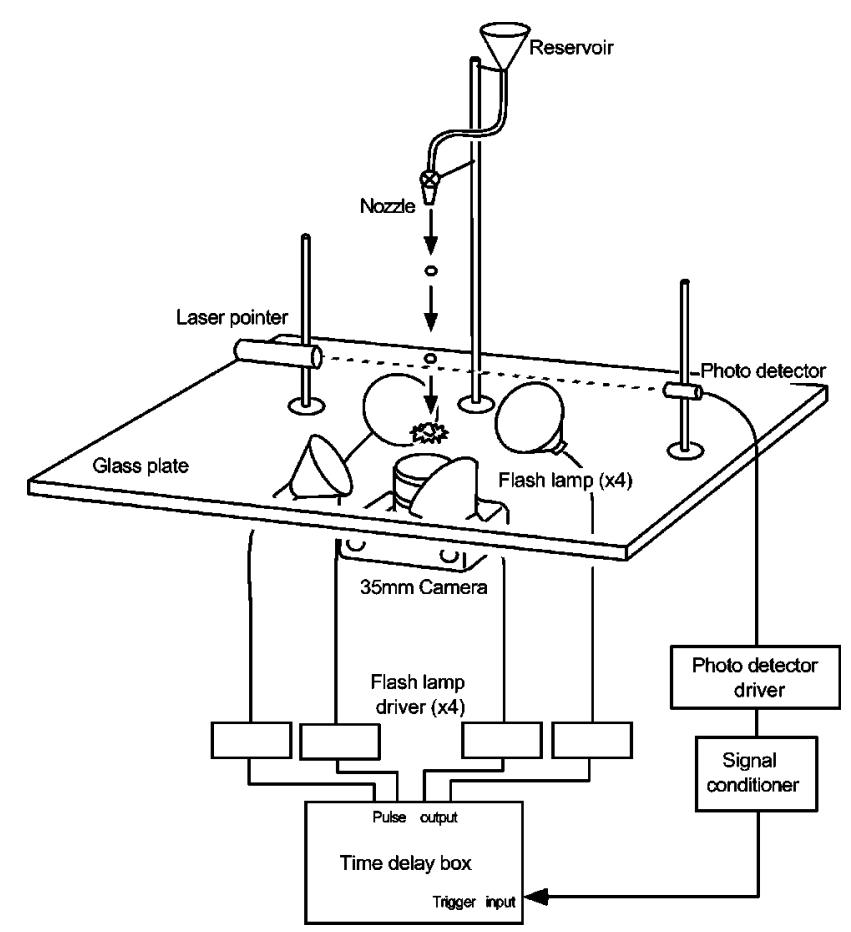

FIG. 2. Experimental setup, showing the relative location of the triggering laser, optical system, and flash lamps. The flash lamps are located under the glass plate.

the trigger sequence used to set off the flashes. We use a simple $5 \mathrm{~mW}$ laser pointer (Radio Shack LX3000) to generate the beam. The homemade triggering box senses the continuous signal coming from the photodiode, generating a sharp jump when the drop blocks the laser light and the sensor signal crosses below a certain threshold. The circuit keeps the signal high for a specified amount of time to avoid generation of spurious flashes. The initial trigger signal is fed into a time-delay box, which after an adjustable time delay $\Delta t_{i}$ triggers the flashes. The digital time-delay box was made by Stanford Research Systems (Model DG535) and has a picosecond resolution for the four separately adjustable trigger output channels. Four separate flash units can in this way

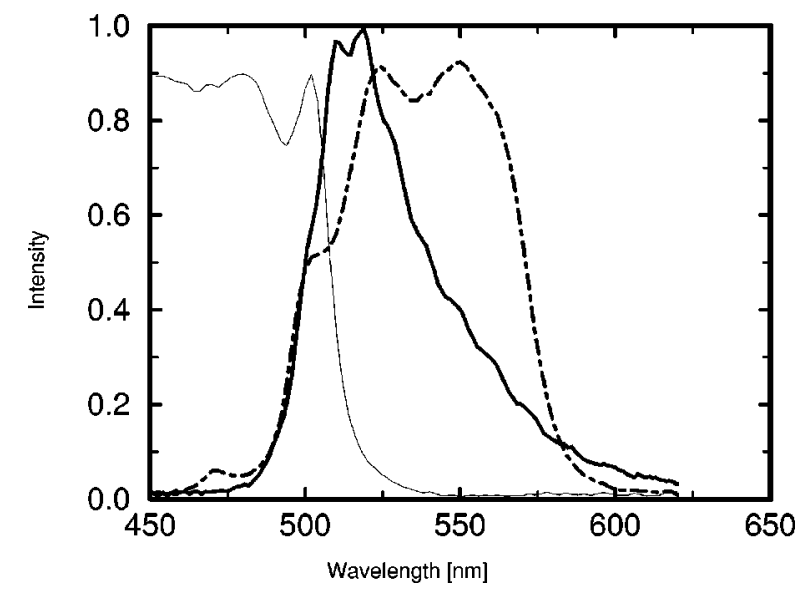

FIG. 3. Transmittance spectra for the blue (thin line) and green (broken line) filters used. The emission spectrum for Fluorescein, in arbitrary units, is also included (thick line). be independently set relative to the initial time. Using the video camera it is fairly simple to determine by iteration the time $t_{0}$ when the drop makes initial contact with the surface. The flash delay times relative to this time $t_{0}$ are denoted by $t_{1}, t_{2}, t_{3}$, and $t_{4}$.

\section{B. Flash lights and optical setup}

We illuminated the impact with a short duration Xenon flash lamps (QuadTech Stroboslave Type 1539-A). The duration of the flash is of the order of $3 \mu$ s (specified by the manufacturer), which totally freezes the motion. Even at the largest impact velocities this corresponds to only about $6 \mu \mathrm{m}$ of travel.

Light reflected and refracted from the fluid surface was often focused close to the fluid edge, interfering with the identification of the contour of the contact line. Imaging the drop through a glass plate also presents some difficulties, such as the generation of uneven background glow, which obscures part of the edge. To remedy this problem we added fluorescent dye to the drop fluid and, in combination with color filters on both the camera and the flash lamps, we were able to photograph the induced fluorescence only. This made identification of the front possible even when using multiple flashes. Each flash lamp was covered with a thick sheet of paper that had a square opening covered by a dichroic blue filter (Edmund Scientific) 5 by $5 \mathrm{~cm}$ in area, which allows only blue and shorter-wavelength light to get through. The camera lens was, on the other hand, fitted with a green filter, concentrated at about the wavelength of the fluorescent light. Figure 3 shows the measured transmittance of the filters used, along with the emission spectrum for the fluorescent dye, i.e., Fluorescein. These were measure using a spectrometer (270 M, Jobin Yvon). The best imaging results were obtained by using very high Fluorescein concentrations of 1 $\mathrm{g} / \mathrm{l}$. This lowered the surface tension of the distilled water to about 50 dyne $/ \mathrm{cm}$, measured with a ring tensiometer. The flash lamps are powerful enough to allow for the use of small apertures for the large depth of field needed, especially for the side views.

The glass was periodically wiped clean with a tissue and alcohol. Lens cleaning paper was also used to wipe the glass plate between drops, until no dust particles were visible.

The edge images taken from the bottom are all in the same plane, i.e., that of the glass plate. Photographs of a ruled template show negligible optical distortions. The relative placement of the flash lamps is shown in Fig. 2. They were placed symmetrically about $20 \mathrm{~cm}$ below the plate, in a flower arrangement around the camera lens. The flashes pointed toward the impact from about $30^{\circ}$ away from the vertical.

The camera was a Nikon F90X with a $105 \mathrm{~mm}$ Nikkor FD microlens, giving a magnification of 1.0 for most of the images. For the largest drop height a second magnification of 0.83 was also used for the largest spreading, as the drop would otherwise extend out of the frame.

The camera aperture is manually kept open in bulb mode during the impact, which takes place in a darkened room. We used both 100 and 400 ASA TMAX black and white film, 


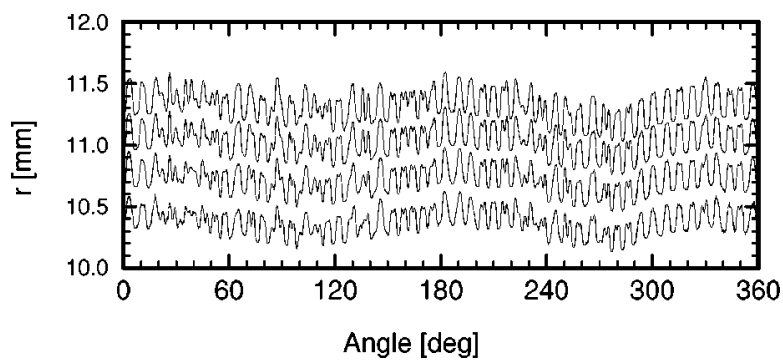

FIG. 4. Typical $r(\theta)$ curves. The flash times are 2.4, 2.6, 2.8, and $3.0 \mathrm{~ms}$ after contact.

with an aperture of $f=5.6-8$ and $11-16$, respectively. The film was developed and scanned into a computer using a Nikon LS-1000 film scanner, with a resolution of approximately 2000 by 2000 pixels over the drop area on the film. Therefore, each digitized pixel corresponds to about $9 \mu \mathrm{m}$.

A monochrome video camera (Sony XC-77) was used for evaluation of spreading rates. It had a $16 \mathrm{~mm}$ microlens, used in combination with extension rings to give the desired magnification. This allowed for the collection of more data points than is practical with film. Here the video signal was simply recorded to a VCR (Panasonic AG-5700) with an open shutter on the camera. The video was then played back frame by frame to the single exposed frame, which was then frozen for measurements from a video monitor. Photographs of typical frozen monitor images are presented in the text.

\section{Edge-detection algorithm}

The edges of the expanding lamella was identified from the scanned intensity fields using a gradient criterion. First, the weight center of intensity of the drop image was identified. Next the pixel-interpolated intensity was calculated along rays extending from this center. The location of the edge was then determined by using maximum gradient. This was repeated, marching azimuthally around the edge over all values of $\theta$ in 4096 steps. Finally, we refined the location of the edge, using a pattern matching algorithm with a template obtained by averaging the intensity profile across a short segment of the edge. To avoid spurious edge detection due to noisy pixels, we enforced the continuity of the radial location $r(\theta)$ within a certain number of pixels. Parametric curve fits were then used for smoothing. The resulting edge was drawn on top of the original image to verify its accuracy. In this way, we constructed the trace of radius versus azimuthal angle $r(\theta)$. Typical resulting curves are shown in Fig. 4. The $r(\theta)$ curve contains a long-wavelength undulation, showing about two cycles around the entire periphery. The deviation is of the same order as the possible stretching of the film or its curvature inside the scanner, but might be due to oscillations of the drop in the air. This variation is only about $1.5 \%$ of the total radius, but was subtracted in the correlation analysis to follow, as it was of the same order as the finger amplitudes.

Three edges could easily be extracted from most of the images. The earliest edge would be the noisiest, due to the superposition of the three successive flashes. It could therefore, in some cases, not be educed from the intensity data.

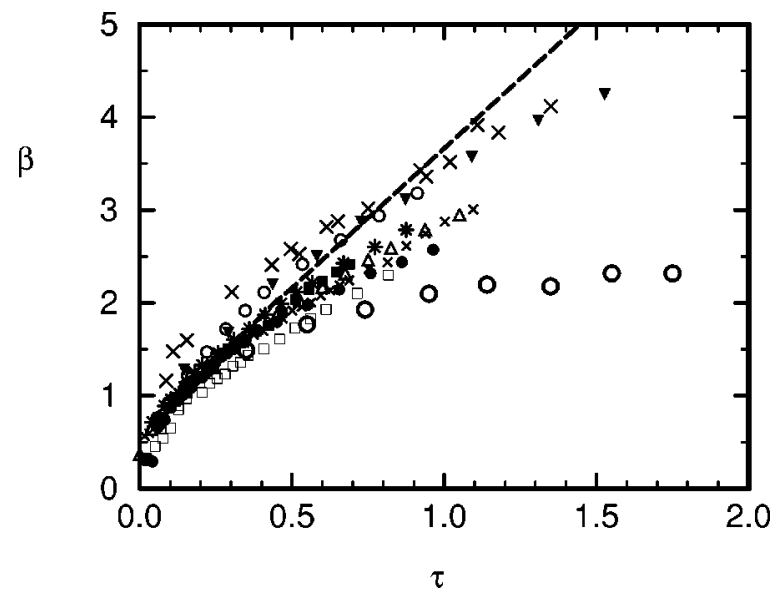

FIG. 5. Nondimensional spreading rates $\beta$ (obtained from video) versus the normalized time for a water drop impacting on an aluminum surface, for a number of different drop release heights, $H(\mathrm{~cm})=6$ (filled circle); 10 (open square); 15 (small $\times$ ); 22 (open triangle); 31 (filled square); 40 (star); 60 (small open circle); 80 (filled triangle); 124 (large $\times$ ); for a water drop with $D=5.5 \mathrm{~mm}$ and $\gamma=60 \mathrm{mN} / \mathrm{m}$. Inviscid calculations without surface tension from Harlow and Shannon ${ }^{14}$ (dashed line); experimental results of Pasandideh-Fard et al. ${ }^{12}$ (their Fig. 5) from much lower Reynolds numbers (large open circle), with $\mathrm{We}=28, U=1 \mathrm{~m} / \mathrm{s}$, and $D=2.0 \mathrm{~mm}$.

\section{RESULTS}

\section{A. Spreading rates}

To verify the efficacy of the experimental approach, we began by measuring the spreading rates of a water drop impacting on a flat metal surface. Those results are in good quantitative agreement with previous studies, as will now be shown. For this part of the study we used a distilled water drop $(D=5.5 \mathrm{~mm})$ with very weak Fluorescein concentrations $(6 \mathrm{mg} / \mathrm{l}$, giving surface tension $\gamma=60 \mathrm{mN} / \mathrm{m})$, and the surface used was anodized aluminum. Figure 5 shows the spreading rates for a number of different drop release heights, thereby varying the impact velocity and impact Reynolds number. These data were obtained using the video system and the spreading was measured directly from a video monitor. Many separate video frames, each taken with one flash, triggered at different time delays $t_{1}$, were used. The use of video allows for the collection of a large number of data points during the spreading and subsequent contraction. Figure 6 shows typical examples of the video frames used for these estimates. The full-resolution video frames resolved the location of the edge better than $1 \%$ of the maximum extent, which is smaller than the variability introduced by the presence of the fingers at the edge.

For the largest drop heights we had to use two flash lamps to determine accurately the time duration from impact. This is necessary, as for the largest drop heights, significant transverse motions of the drop are induced, most likely due to the unsteady air flow created around it during the fall. Any sideways motions will cause the drop to cut the laser beam at slightly different horizontal locations relative to the drop center. This also induces vertical shift in drop location as it cuts the beam, thus changing $t_{0}$. This can be corrected for by triggering a first flash while the drop is in the air, to fix $t_{0}$ and a second flash during the impact, to get the spreading 


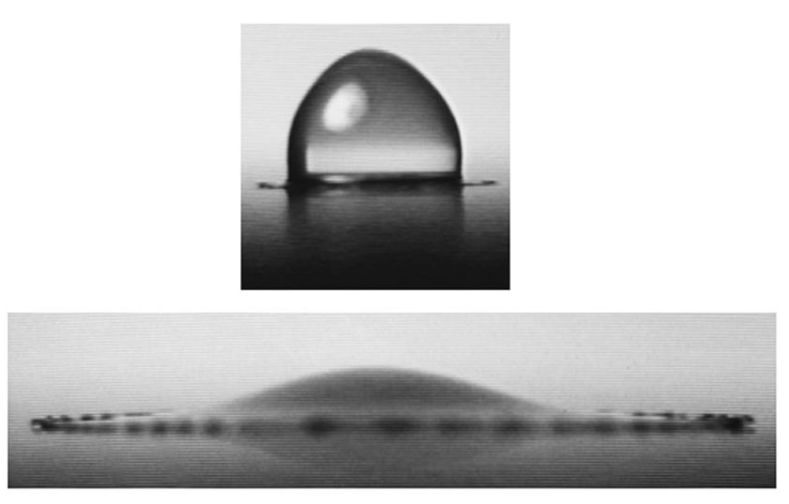

FIG. 6. Typical video frames used to estimate spreading rates.

relative to this corrected $t_{0}$. Figure 7 shows a video image with such a double exposure.

The spreading for a number of release heights $H$ are included in Fig. 5. The spreading diameter $(\beta=2 r / D)$ is normalized by the equivalent spherical drop diameter $D$ and the spreading time by impact velocity $U$ and this diameter, i.e., $\tau=t U / D$. We have also included the spreading rates from the inviscid calculations of Harlow and Shannon ${ }^{14}$ and those from Pasandideh-Fard et al., ${ }^{12}$ measured photographically for a considerably lower Reynolds number. The rates obtained with our system agree well with these previous results. There is, however, a systematic increase in spreading rates versus impact $\mathrm{Re}_{D}$. Similar increase in spreading rates with $\operatorname{Re}_{D}$ were also observed by Fukai et al., ${ }^{16}$ from experiments and computations of the impact of a tin droplet.

For the highest impact velocities, the initial outwards velocity of the lamella exceeds the impact velocity. Harlow and Shannon ${ }^{14}$ suggest that there should be a universal spreading curve for very large Reynolds numbers, i.e., for negligible viscous effects. Their asymptote has a value of 1.6 times the impact velocity. Our data exceed this value immediately after the impact. Other researchers have also noticed this. ${ }^{1}$

One should note that, in the presence of fingers, the location of the tip of the lamella obtained from side views, will, in general, give the maximum extent of the front, not the average radius.

The results for different drop height $H$ portray rather different spreading rate curves, rather than showing a gradual

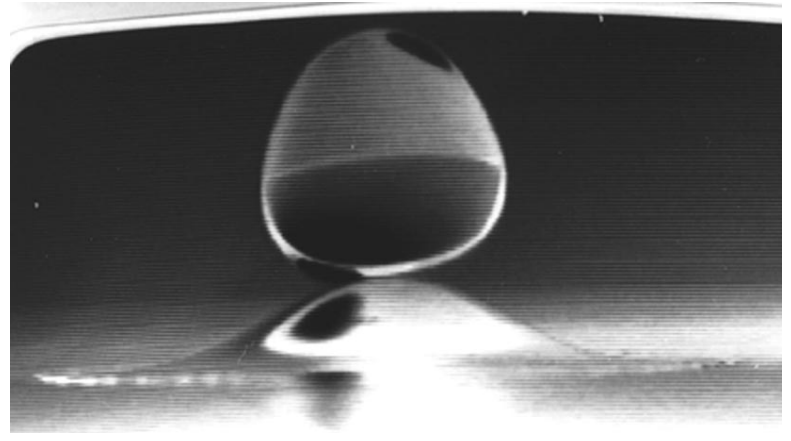

FIG. 7. A video frame showing the double flash, used for the accurate determination of impact times for the largest drop heights.
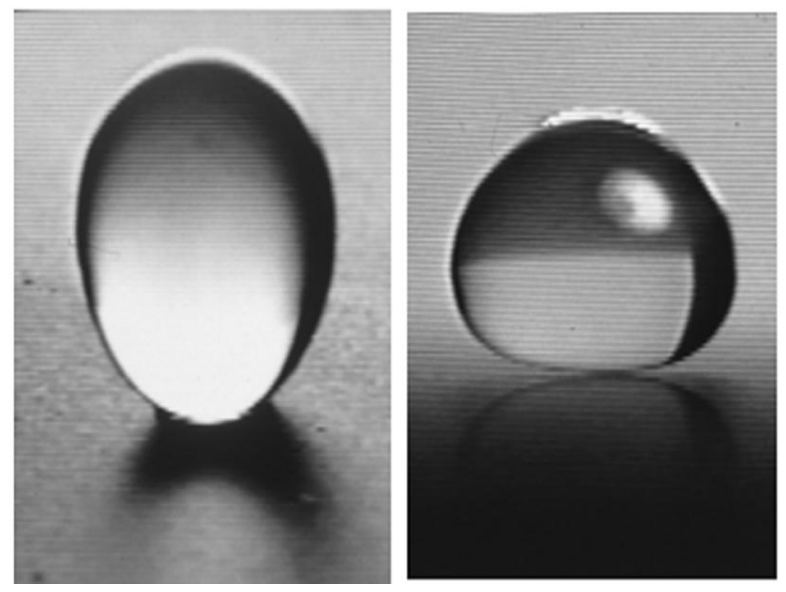

FIG. 8. Video frames selected to demonstrate the deformation of the drops during the fall. The two frames are from different perspectives and are not to the same scale.

change in shape. One should keep in mind that for these high Weber numbers the drops do not reach spherical shape, but are either oblate or prolate at impact, as is clearly seen in select video frames shown in Fig. 8. This shape can be expected to be about the same for a fixed drop height $H$, as the drop is at the same stage in its oscillation, but will be quite different for widely different values of $H$. Aesthetically one would prefer a spherical drop, but in this high Webernumber regime studied here, some variability in the shape has to be accepted. Mercury drops can be used to achieve the same Reynolds number, while retaining their spherical shape, due to the much higher surface tension and lower kinematic viscosity. ${ }^{2,3}$ The Weber number for the $4.8 \mathrm{~mm}$ Mercury drop used by Worthington was 212 . The direct effects of this nonsphericity were not studied here.

Figure 9 shows the longer-time spreading evolution of the front for one release height $H=20.4 \mathrm{~cm}$, which includes both the expansion and subsequent contraction. The data during the contraction phase are less repeatable than those during the expansion, as the capillary waves on the lamella during the contraction are highly dependent on the specifics of

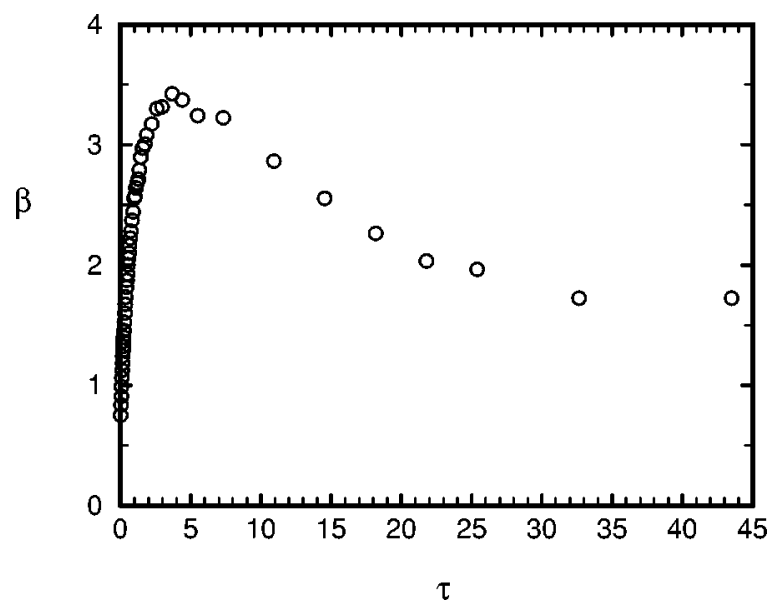

FIG. 9. The spreading and subsequent contraction for a drop released from a height of $20.4 \mathrm{~cm}$ onto anodized aluminum surface. Drop diameter $D$ $=5.5 \mathrm{~mm}$. 


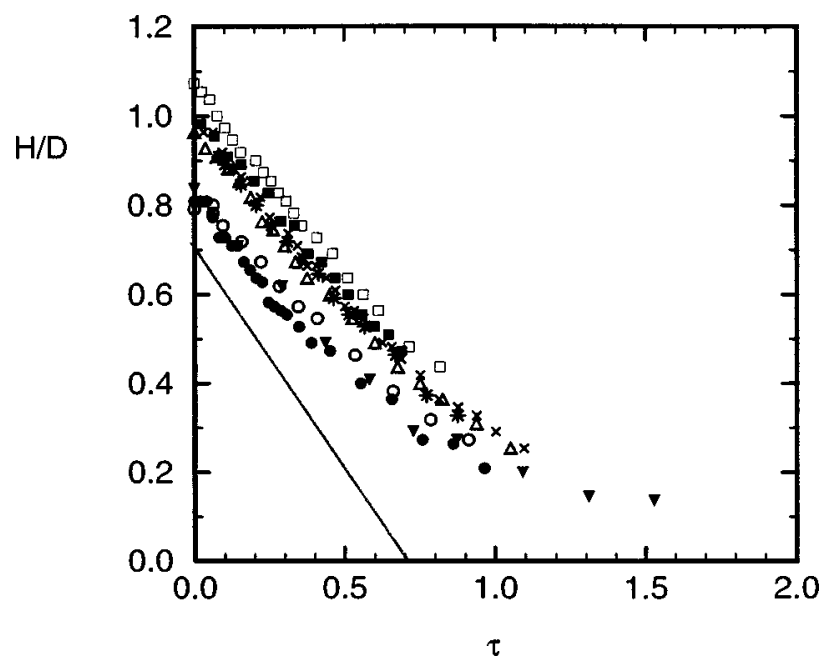

FIG. 10. The vertical compression of the drop for a number of released heights. The same symbols as in Fig. 5. The solid line indicates the motion in freefall, i.e., if the top of the drop were not to feel the presence of the solid surface.

the frontal shapes, adding a random component to the process. This figure shows clearly the large difference in the time scales between the spreading and subsequent contraction. The capillary patterns on the receding surface of the drop are shown in a later section. The maximum spreading diameter reaches a value of 3.5. The model in Ref. 12 [their Eq. (18)] predicts a somewhat larger maximum spreading of 4.5 , when using the following values for the impact shown in Fig. 9, i.e., $\mathrm{We}=\rho D U^{2} / \gamma=363, \mathrm{Re}=U D / \nu=11000$, and advancing contact angle $\theta_{a}=110^{\circ}$.

The height of the drop during the impact is plotted in Fig. 10. These values have been normalized by the spherical drop diameter, thus giving an indication of the deformation of the drops in flight. The diameters are as much as $20 \%$ off the spherical diameters. The slowdown is rather insignificant during most of the spreading time, as is shown by the comparison with the freefall impact velocity, drawn as a line.

We conclude that our spreading rates are in good quantitative agreement with previous studies.

\section{Drop impacting on a glass plate}

In what follows we will concentrate on drops impacting on a glass plate, which allows for observations through the surface. These impacts were studied in detail using photographic film for one release height of $H=50$, with a few data for $H=28.8$ and $80 \mathrm{~cm}$. The spreading rates for a drop of diameter $5.2 \mathrm{~mm}$ and released from $H=50 \mathrm{~cm}(\mathrm{We}=1020$; $\mathrm{Re}=16300)$ are shown in Fig. 11. Here the average radii were calculated from the edges traced from the films, giving a very smooth result. The figure includes results from 75 separate edges, many of which are indistinguishable in the graph. The model referred to above predicts a maximum spreading of $\beta=5.5$ (using $\theta_{a}=27^{\circ}$ ), which represents an excellent asymptote for the data. This value of the advancing contact angle was taken from Pasandideh-Fard et al. ${ }^{12}$ (their Table I).

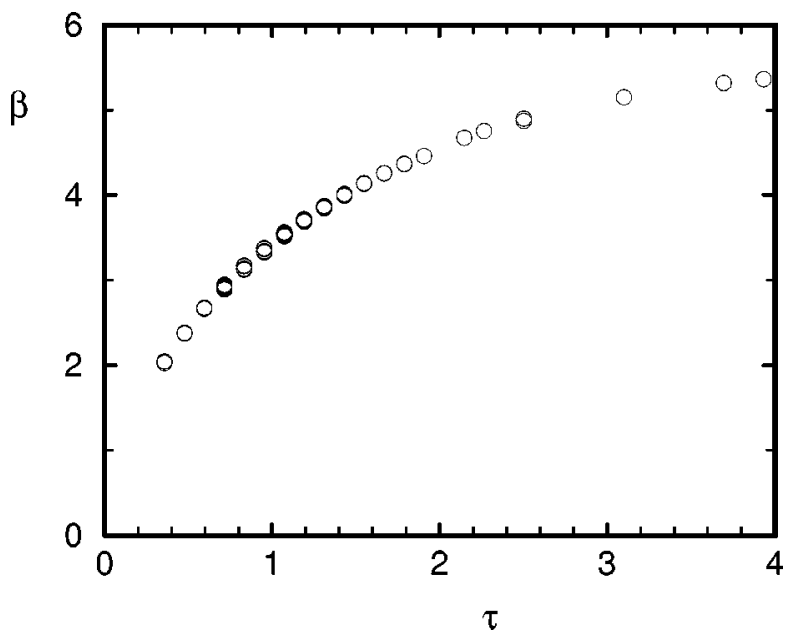

FIG. 11. The normalized spreading of a drop released from $H=50 \mathrm{~cm}$, impacting a glass plate. The spreading was measured using film. Here $D$ $=5.2 \mathrm{~mm}$ and $\gamma=50 \mathrm{mN} / \mathrm{m}$.

\section{No splashing}

No splashing occurred during these impacts. Mundo et al. ${ }^{10}$ have studied the splashing threshold in terms of the Ohnesorge number $\mathrm{Oh}=\mu / \sqrt{\rho \gamma D}$. The value we get is $\mathrm{Oh}$ $=2 \times 10^{-3}$ for the $\operatorname{Re}_{D}=16000$ case. These values put us close to the boundary between the splashing and nonsplashing regimes, in their Fig. 13 (their p. 162), but slightly inside the splashing side. It is, however, stated in their paper, on p. 162 , that the theoretical correlation breaks down for $\operatorname{Re}_{D}$ $>2000$. The very smooth float-glass surface we used may also explain our lack of splashing. The curvature of the surface used by Mundo et al. may also play a role. There is therefore no obvious disagreement between the two studies.

\section{B. Fundamental wavelength}

By studying the fundamental fingering wavelength and radial evolution of the frontal undulations, one can hope to illuminate the nature of the fingering instability and pinpoint its origin.

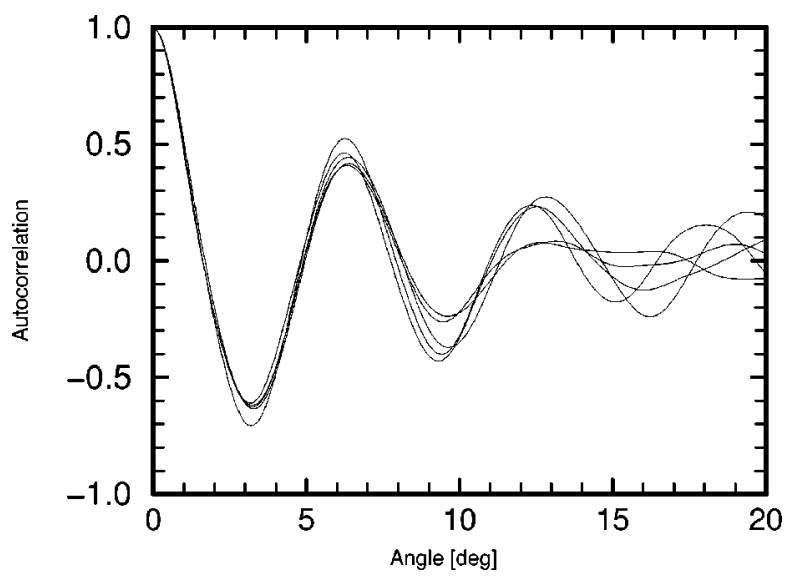

FIG. 12. The autocorrelations of frontal undulations for $H=50 \mathrm{~cm}$ at $t_{1}$ $=1.8 \mathrm{~ms}$ after impact. Results from five separate drops are included. 


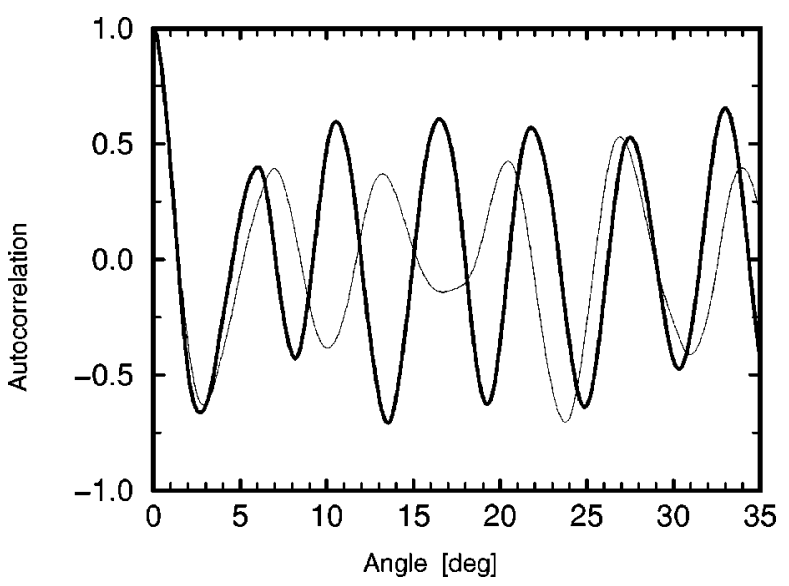

FIG. 13. The autocorrelations of frontal undulations for $\beta=3.7$. The two curves correspond to the two outermost edge segments shown in Fig. 20.

The wavelength was first studied using autocorrelations. The long-wave undulations described in Sec. II C were first subtracted. We have thus assumed that the two are driven by different physical mechanisms, justifying the study of the fingers using the correlation on the smaller wavelengths only. The longer wavelength is most likely associated with the fundamental mode of free oscillations of the drop in the transverse direction during the fall. Figure 12 shows some typical autocorrelations of the frontal undulations. The angular distance to the first shifted peak is selected here as a measure of the characteristic angular size of these undulations. In Fig. 14 the angle of this maximum has been converted into the corresponding characteristic number of fingers around the periphery, i.e., $N_{f}=360^{\circ} / \theta_{\max }$.

The characteristic wavelength does not change substantially as the front advances. There is, however, a weak reduction in the number of fingers, which will be discussed in later sections. This is consistent with Loehr's ${ }^{6}$ results, who observed qualitatively that the number of fingers remained unchanged during the spreading. We also counted azimuthal undulation from video images (taken from above at an angle) of the water drops impacting the aluminum plate. Keeping in

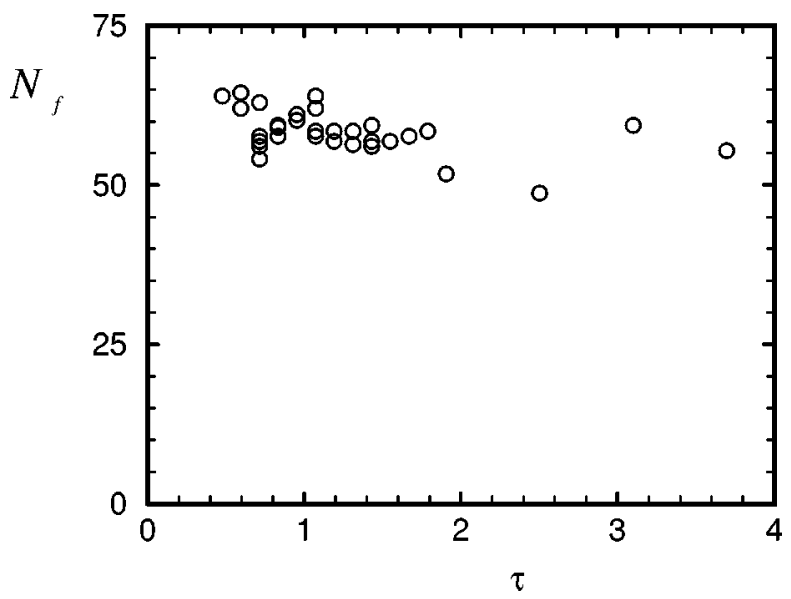

FIG. 14. The number of fingers at the edge during the expansion. Here $H$ $=50 \mathrm{~cm}, D=5.2 \mathrm{~mm}$.

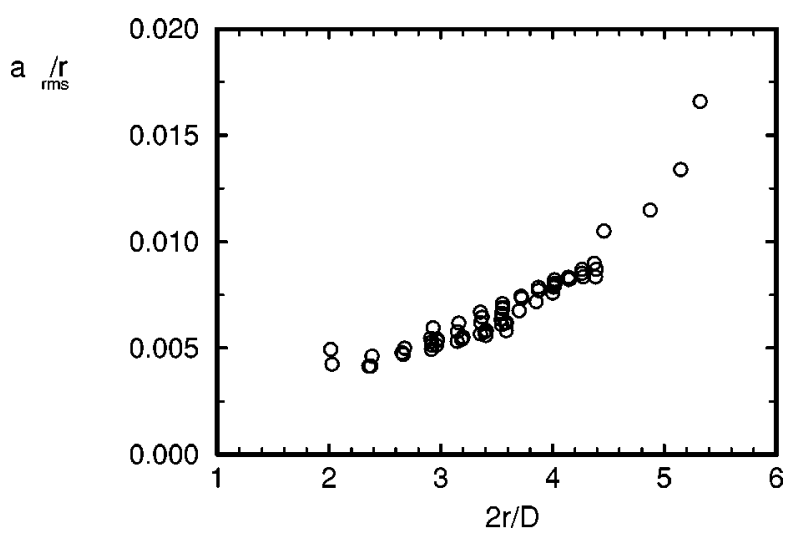

FIG. 15. The growth in the rms amplitude of the frontal undulations normalized by the local radius, during the spreading of the drop, for $H$ $=50 \mathrm{~cm}$ and $D=5.2 \mathrm{~mm}$.

mind the subjective element in such counting, the number of fingers remained fixed during the expansion.

The amplitude of the secondary autocorrelation peaks increases substantially as the expansion nears the end, as is shown in Fig. 13 for $\beta=3.7$. Here the correlation persists at high values for many cycles, indicating an increased azimuthal coherence, as capillary forces become more significant.

The number of fingers, at a fixed radial location, changes clearly between the three different drop release heights. Increasing the impact velocity increases the number of fingers, as expected. ${ }^{1,7}$ The number of fingers for $\beta=2$ is approximately 51, 55, and 66 for drop heights of $28.8,50$, and 80 $\mathrm{cm}$, respectively.

Being faced with the unusual situation of having a naturally periodic function the complication of edge windowing can be avoided when taking the Fourier transform of this edge data. This was done but the results (not shown here) gave peaks in the spectra that were quite broad. This broadness is due to the lack in phase coherence around the drop, as will be discussed in a later section. The use of Fourier transforms to characterize the frontal shapes is therefore somewhat dubious and the lack of a clear peak makes the autocorrelations better suited to study the characteristic azimuthal length scales.

\section{The amplitude of the frontal undulations}

An important characterization of instabilities are their growth rates. The mechanism proposed by Allen would, for example, be characterized by an exponential growth of the amplitude of the frontal undulations starting as the front decelerates. The varying rate of deceleration would, however, complicate this prediction.

Here we can calculate the rms amplitude of the frontal undulations from the photographs and investigate how they grow during the spreading. This is done in Fig. 15. Here the undulations are normalized by the local mean spreading radius. The long-wave undulations described earlier were first subtracted from the $r(\theta)$ curves. It is clear that the normalized amplitude grows initially approximately linearly between a $\beta$ of 2.4 and 4.6. If one extrapolates this straight line 

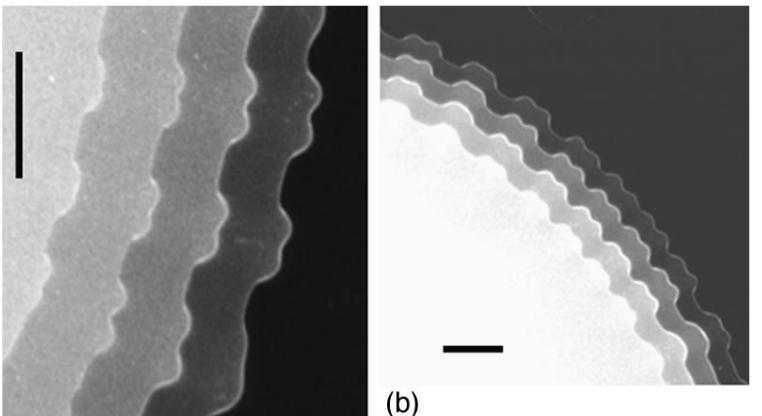

(a)

(b)
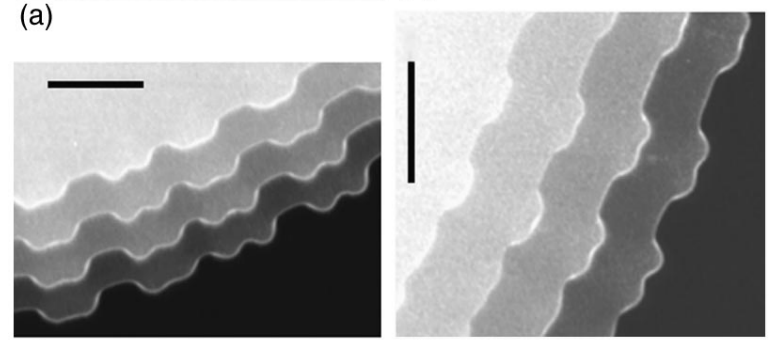

(c)

(d)

FIG. 16. Some typical examples of the splitting of fingers during the expansion. Flash spacing is $\Delta t=0.2 \mathrm{~ms}$ in all panels. The first flash in panels (a) and (d) are $1.2 \mathrm{~ms}$ after contact and in (b) and (c) $1.8 \mathrm{~ms}$ after initial contact. The scale bars are $1 \mathrm{~mm}$ long.

toward zero the undulations will vanish at a radius of approximately $1 \mathrm{~mm}$. However, the data points at $\beta=2 r / D$ $=2.0$ appear to fall higher than this line, consistent with our later discussions of the instability mechanism.

The amplitude grows rapidly as the drop approaches the maximum spreading. This occurs when the mean front has come to a halt, whereas only the fingertips keep spreading radially; see Fig. 22.

\section{RADIAL EVOLUTION OF THE FRONTAL SHAPES}

By triggering the four flashlamps at slightly different instants, one can study the temporal evolution of the frontal shape during the spreading, as is demonstrated in Fig. 1. Numerous photographs were taken with various timing combinations of the four flashes. In this way both the short- and long-term evolution can be studied.

Figure 16 shows a closeup of typical photographs used for this purpose. The clarity of these photographs leaves no ambiguity as to where the front is located.

The development of the finger shapes can be studied in many different ways. From visual observations of many realizations clear patterns emerge. We describe and show examples of these evolution patterns, as well as try to characterize how frequently they occur.

\section{A. Splitting of fingers}

The large azimuthal variations in shapes make taking the average of finger shapes blur the actual evolution of individual fingers. Identifying by eye which evolution is most common or significant has, on the other hand, the disadvantage of being very subjective. Here we follow a hybrid technique to educe the most representative finger shape. It proceeds in the following manner: First, a program finds the

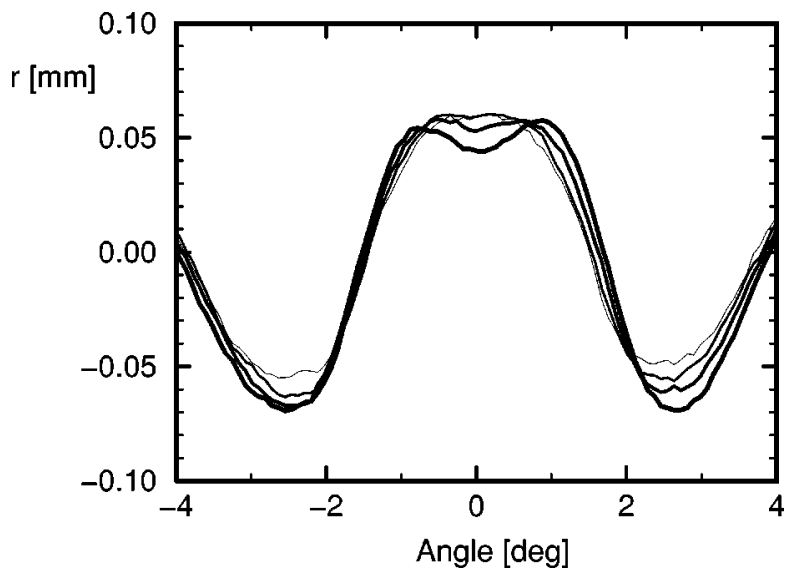

FIG. 17. The "median" shape of the fingers for four radial locations. The thickness of the lines shows the progression in time, with $\Delta t=0.2 \mathrm{~ms}$. The earliest shape corresponds to $t_{1}=1.2 \mathrm{~ms}$.

mean finger shape for a particular edge, by identifying each finger by its tip. All of the pronounced fingers are then shifted together and the average shape found. In a second sweep, the 20 fingers having the smallest least-square deviation from this mean are then selected and a new mean calculated from the average of those fingers only. Finally, the finger closest to this second mean is selected as, in some sense, being the most representative finger shape. The radial evolution of this finger is then studied. The results obtained in this way confirm what is visually observed i.e., the finger widens and its tip splits developing two peaks. Figure 17 shows the beginning of this evolution. As is clear by the examples shown in Fig. 16, there is considerable variability in shapes, as the drop edges undergo this splitting. This depends particularly on how close the adjacent fingers are to each other. In Fig. 16(b), where the fingers are closely spaced, the two peaks on each finger become equal in amplitude. This is the precursor of the merging described in the following section.

The speed with which the fingers widen were obtained from these figures and compared to theoretical values for capillary waves, as is explained below.

\section{B. Merging of fingers}

This characteristic splitting of the original fingers is frequently followed by a merging of adjacent fingers, in a fashion where two adjacent double fingers combine to form three larger and more pronounced fingers. Some examples of this are shown in Figs. 18-21. The deepest trough transforms in this way into the largest crest. When this merging occurs in isolation, sufficiently far away from the adjacent bumps, the remaining side bumps grow and travel sharply away from the center finger.

The prevalence of these merging interactions are difficult to determine by three or four snapshots, as one has to catch the progression of events at least at two identifiable instances in these interactions. They do not occur at a fixed radial location, but depend crucially on the uneven azimuthal separation of adjacent fingers. A cursory observation of Fig. 1 demonstrates this difficulty. One can argue that approxi- 


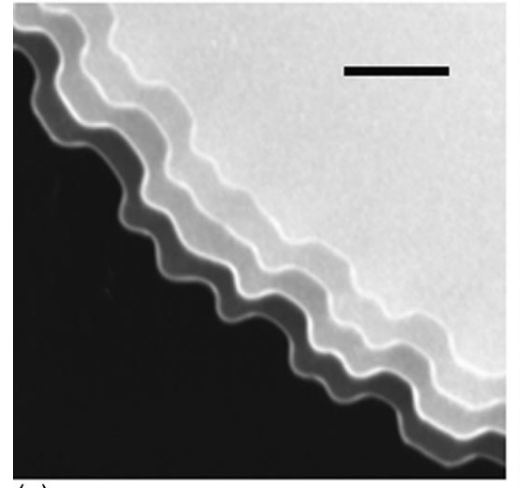

(a)

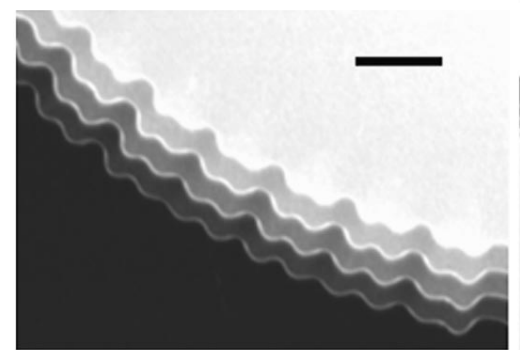

(b)

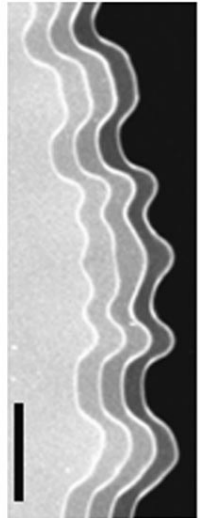

(c)

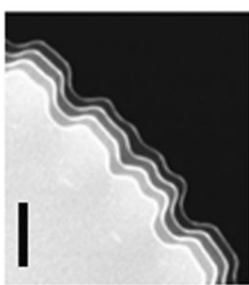

(d)
FIG. 18. Some typical examples of the merging of fingers during the expansion. The flash separation is $0.2 \mathrm{~ms}$ in all panels. The first flash is at 2.4 $\mathrm{ms}$ after initial contact in (a) and (b), $3.0 \mathrm{~ms}$ in (c), and $3.6 \mathrm{~ms}$ in (d). The scale bars are $1 \mathrm{~mm}$ long.

mately 29 merging events can be identified in the figure. These are in various stages of evolution, but this number corresponds to about half of all the fingers.

The speed with which the merging occurs can be estimated from images where adjacent fingers are caught in the act. This can be displayed in terms of capillary phase speed. They should take about the same amount of capillary time, irrespective of the spreading velocity. This means that for merging at small radii it should last over a longer range of $r$, whereas later in the spreading the merging should take place in shorter variations in the radius. This is indeed evident in the various panels of Figs. 18-21, where the merging event takes place in a radial distance corresponding to somewhere between one and four fundamental azimuthal wavelengths.

Figure 22 shows the motion of the contact line at maximum extent. It demonstrates how the isolated fingers continue moving, while the valley between them has stopped. These continue to produce the pronounced fingers left behind when a drop of ink lands on a piece of paper. ${ }^{7}$ When two fingers are too close together, the contact line between them moves remarkably similar to the natural oscillations of a string. Here the two fingers, however, combine on the upswing, to form one finger. For water on glass, these fingers are pulled back and do not leave a secondary ring of drops, as Worthington observed for a drop of mercury impacting on glass.

\section{Phase speed of capillary waves on the lamella}

Figure 1 shows that the wave form is not coherent around the entire drop, but rather displays disparate sections

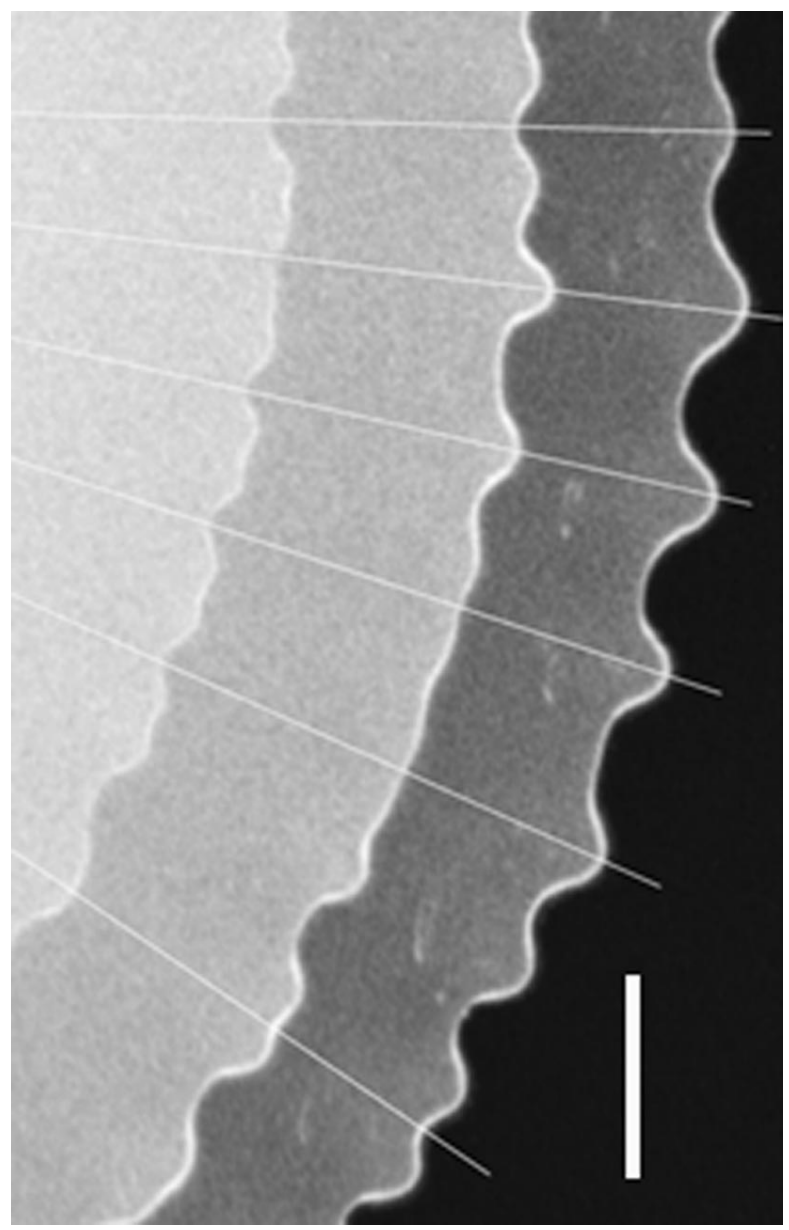

FIG. 19. Some more examples of the merging of fingers during the expansion. Drop release height is here $H=28.8 \mathrm{~cm}$ with the three flashes at 2.0, 2.6, and $3.2 \mathrm{~ms}$ after initial contact. The scale bar is $1 \mathrm{~mm}$ long. Lines have been drawn from the center of the drop to guide the eye.
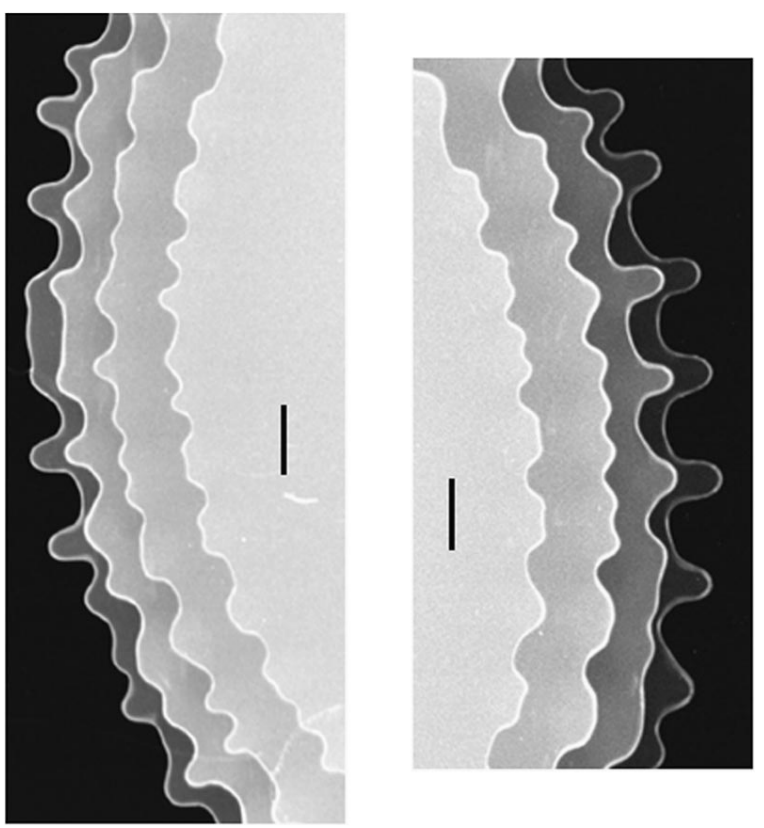

FIG. 20. The rapid evolution the frontal shapes as the drop edge nears maximum extent. The time separation of the flashes was $1.0 \mathrm{~ms}$, with the first flash at $3.2 \mathrm{~ms}$ after contact. The two panels show two sides of the same drop. The scale bars are $1 \mathrm{~mm}$ long. 
with fixed periodicity. There is no clear reason to expect the waveform to be in phase around the entire drop, unless the initial disturbances are exactly azimuthally periodic, or if information travels azimuthally around the drop during the expansion. It is therefore instructive to estimate the azimuthal speed of the frontal disturbances. Since gravity is negligible during most of the expansion, the only plausible candidate for driving these motions are capillary forces. Loehr and Lasek ${ }^{5}$ have put forth a similar speculation.

The speed of these disturbances can be estimated from the dispersion relation for capillary waves on a shallow layer of fluid, which is given by Lighthill, ${ }^{19}$

$$
c^{2}=\left(g+\frac{\gamma k^{2}}{\rho}\right) \frac{\tanh (k h)}{k},
$$

where $k$ is the wave number, $c$ is the phase speed, and $h$ is the depth of the fluid layer. For a $1 \mathrm{~mm}$ long wave on water, the second term inside the parentheses is 200 times larger then the first, justifying one in neglecting gravity from this expression. Viscous effects have also been ignored in this relationship.

One can obtain a rough estimate of the thickness of the lamella from the edge-on video images. For the largest impact Reynolds numbers this suggests a thickness of the initial jet of about 0.1-0.3 $\mathrm{mm}$. During the end of the expansion the edge has thickened up to as much as $1 \mathrm{~mm}$. The thickness of the boundary layer under the fingers can be estimated on dimensional grounds as $\delta=\sqrt{\nu T}$, where $T$ is the time from

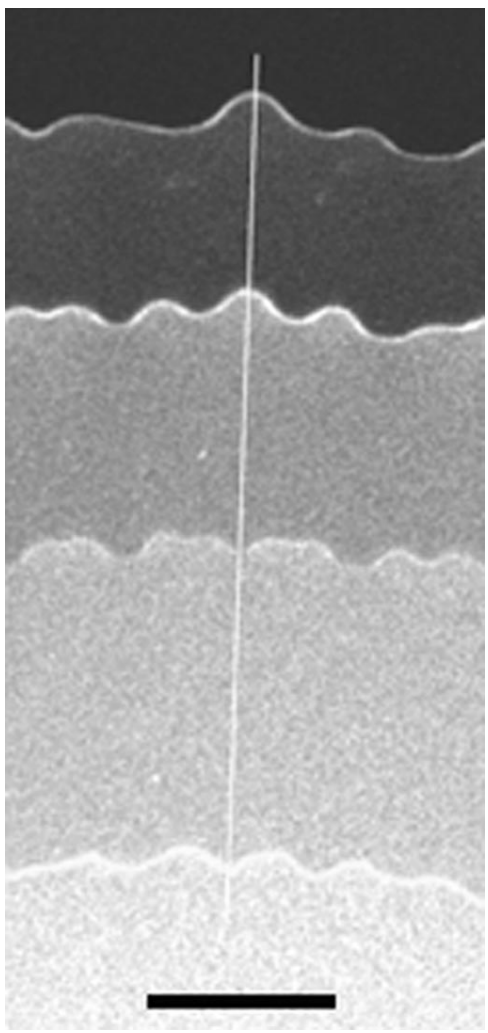

FIG. 21. Sequence of edges showing the whole process of formation of two peaks on each finger and the subsequent merging into three fingers. The time separation is $\Delta t=0.5 \mathrm{~ms}$ with the first flash at $0.2 \mathrm{~ms}$ after contact. The scale bar is $1 \mathrm{~mm}$ long. The first edge has been enhanced.
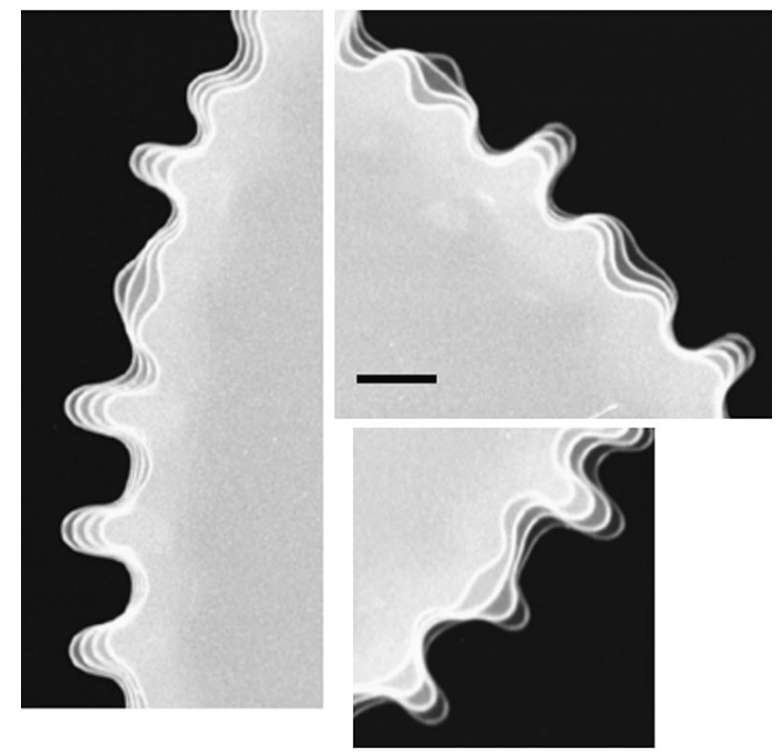

FIG. 22. The changes in the frontal undulations at maximum extent. Here $\Delta t=0.3 \mathrm{~ms}$, with the first flash at $5.7 \mathrm{~ms}$ after contact. The panels are all to the same scale and the bar is $1 \mathrm{~mm}$ long.

initial contact with the surface. For an expansion time of 6 $\mathrm{ms}$ this give $0.25 \mathrm{~mm}$, which is quite consistent.

With these estimates of $h$ the phase speed of capillary waves of different wavelengths is shown in Fig. 23(a). Figure 23(b) shows the ratio of the capillary phase speed of the characteristic wavelength of the fingers, $k_{f}=2 \pi / \lambda_{f}=N_{f} / r$, to the spreading velocity of the lamella. The spreading velocity was estimated using the data shown in Fig. 11. Two competing factors affect this ratio: First, the spreading velocity slows down due to viscous forces, rapidly approaching zero at maximum extent. Second, the length of the periphery increases, making the fundamental wavelength longer. The layer thickness $h$ is also changing, but unknown. The results for a range of depths, enveloping likely values of $h$, are included in the figure. The phase speed associated with the widening and splitting of the fingers, shown in Figs. 16 and 17 , can be estimated by the angular location of the zero crossing, or mean amplitude of the shape, as well as by the motion of the two peaks after they form. This speed is indeed rather slow, having $c=5 \mathrm{~cm} / \mathrm{s}$ for the zero-crossing data in Fig. 17. The splitting velocity of the double peak at the finger tip is significantly larger at $20 \mathrm{~cm} / \mathrm{s}$. These values are within plausible values for this velocity in Fig. 23(a).

Figure 23 shows that early in the spreading the speed of the capillary waves is of the order of $15 \%$ of the expansion velocity. During the later part of the spreading the sheet has become so deep at the edge that the $h \approx \infty$ limit can be used. Using this limit, the capillary phase velocity reaches half of the expansion velocity only when the expansion is at $\beta$ $=4.8$, i.e., at about $85 \%$ of maximum extent.

It is clear from this graph that only at the end of the spreading do capillary effects become dominant, in agreement with Loehr and Lasek. ${ }^{5}$

Figures 24 and 25 show a few examples of what we speculate is a localized disturbance, probably caused by a dust particle on the glass, which initiates a "shock wave," 

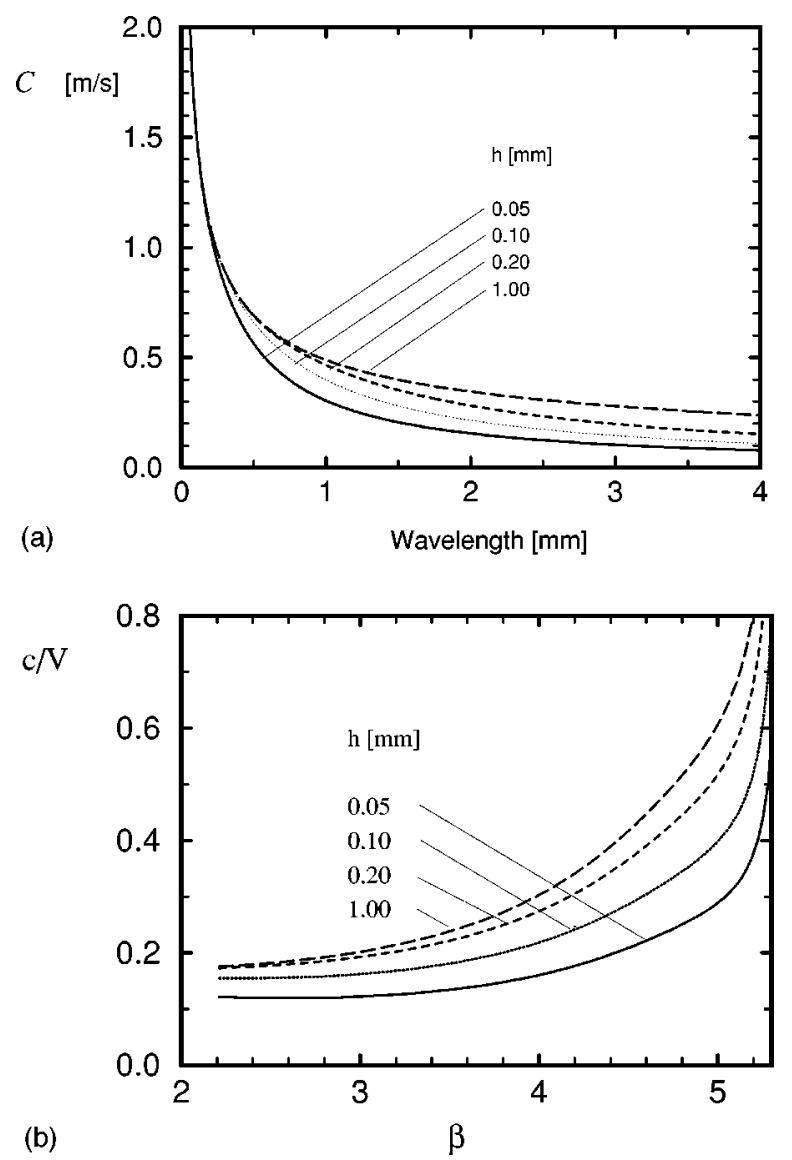

FIG. 23. (a) The speed of capillary waves on thin sheets of water. (b) The speed of capillary waves normalized by the drop spreading velocity, for a lamellar thickness of $0.05-1.0 \mathrm{~mm}$ and surface tension of 50 dynes $/ \mathrm{cm}$. The expansion velocity is taken from the data in Fig. 11.

spreading outward at maximum speed. The angle of this disturbance cone, drawn in Fig. 25, is significantly wider than the local azimuthal spreading due to the radial increase in circumference. This is indicated by radial lines drawn from the drop center that are also shown in Fig. 25.

The maximum azimuthal phase velocity $v_{\theta}$ measured from this image is $1.2 \mathrm{~m} / \mathrm{s}$ for this particular shock. By making the assumption that the smallest allowable wavelength is the same as the layer depth, using Eq. (1), this velocity translates into an effective lamellar depth of $0.21 \mathrm{~mm}$, which is in very good agreement with our other estimates of the depth. The dynamical changes in the frontal shape are therefore local in nature and cannot travel far along the front in the azimuthal direction. The rapid falloff in the autocorrelation presented earlier seems to further support the local nature of the interactions.

\section{Initiation of the azimuthal disturbances}

Video frames taken immediately after the first contact show clear azimuthal unevenness in light intensity at the contact line. Some examples of this are shown in Fig. 26 for $H=60 \mathrm{~cm}$. This is true even when the contacting fluid only covers about a quarter of the drop diameter, in Fig. 26(a).

The bright radial lines extending from the front are probably artifacts from the caustics due to light coming through

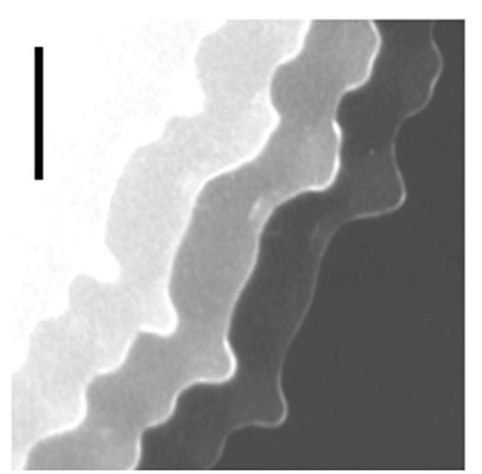

(a)

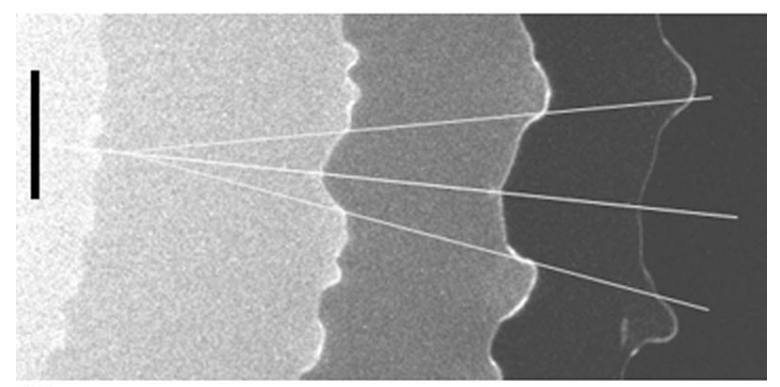

(b)

FIG. 24. Examples of large-amplitude "shock"-type disturbances at the expanding contact line. (a) Flash times are 1.2, 1.4, 1.6, and $1.8 \mathrm{~ms}$ after initial contact. (b) Flash times $0.2,0.7,1.2$, and $1.7 \mathrm{~ms}$ after initial contact. The scale bars are $1 \mathrm{~mm}$ long.

the drop, interacting with the undulations on the front. No filters were used in this part to reduce those reflections. They might, however, not be due to undulations at the front, but rather due to crimpling of the surface at the crease between the drop and the outgoing jet. A double-pulse image in Fig. 26(d) seems to show a smooth crease once the jet has traveled from underneath the drop, supporting the former, but these images are inconclusive as to where these azimuthal undulations reside or to what their shape is. However, their presence at very early times is clear. Better resolved images are needed here.

One could speculate that the initial contact takes place along a ring, thus trapping air in the center. This ring of fluid that touches the solid surface first, might come down too fast to push away evenly the air under it, developing the seeds for the azimuthal instability by a Rayleigh-Taylor instability as the rapidly decelerating ring of fluid touches the surface. This is essentially the same instability mechanism as suggested by Allen, ${ }^{8}$ except it takes place before the jet begins and is not due to the viscous deceleration of the jet itself, as he suggests.

The presence of the azimuthal disturbances close to the initial impact is in agreement with Loehr's contention that the fingers begin early on in the spreading.

The formation of the air bubble at the center of the fluid contact is also quite clear in these pictures. For the earliest video images [Figs. 26(a) and 26(b)] the bubble is missing, but there is a darker irregular region around the center. Figure 27 shows an enhanced image of this region. This is presumably the air layer caught under the drop, which is in the 


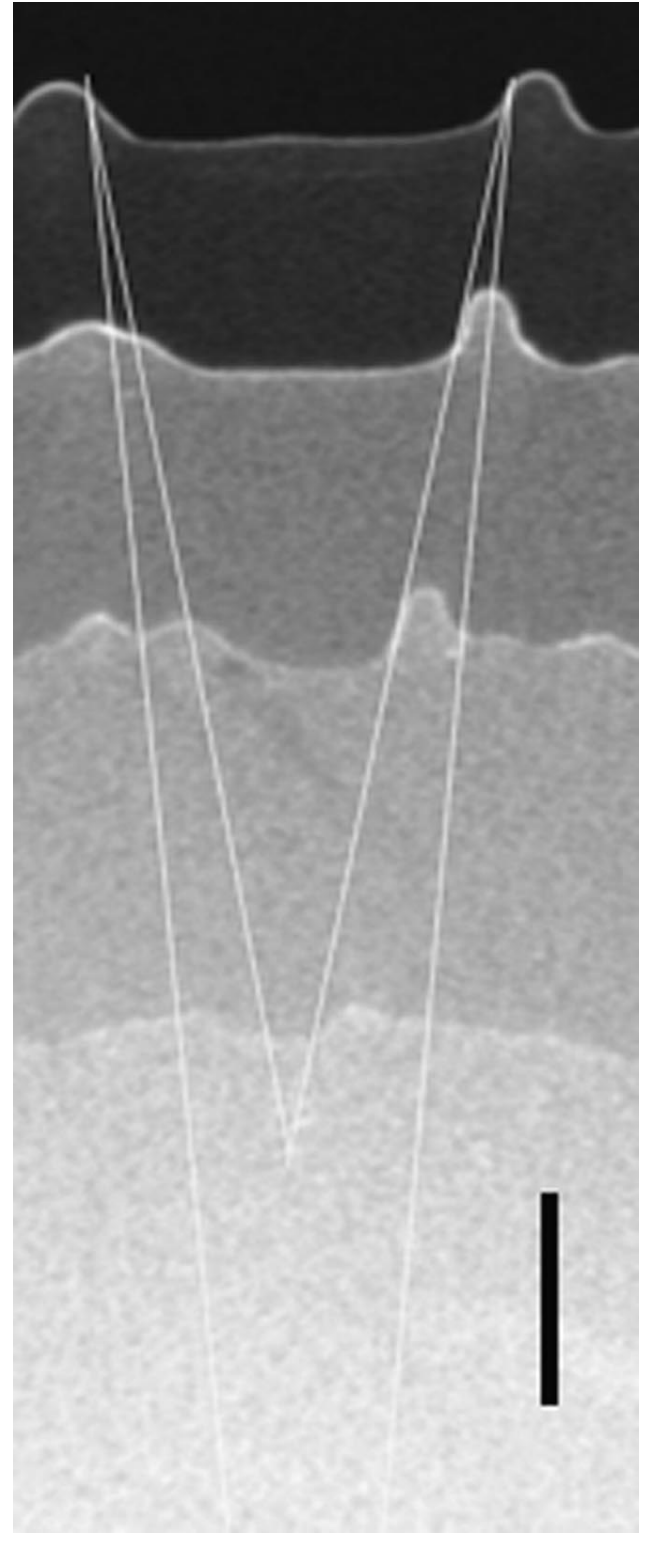

FIG. 25. The "shock-type" capillary wave. The outer lines are drawn from the center of the impact and the inner ones mark the extent of the disturbance. The four flashes are spaced by $0.5 \mathrm{~ms}$. The radius at the last flash is $10.32 \mathrm{~mm}$. The azimuthal phase velocity of the disturbance is $1.24 \mathrm{~m} / \mathrm{s}$. The scale bar is $1 \mathrm{~mm}$ long.

process of pulling together by the surface tension to form the bubble. It has already formed when the drop contact line with the jet is only about two-thirds of the drop diameter, in Fig. 26(c). This bubble has been beautifully photographed by Chandra and Avedisian. ${ }^{11}$ They make a convincing argument that it is formed from air trapped under the drop and not due to cavitation due to decompression of the liquid. They also show how this bubble rises up through the drop. However, at our much higher impact Reynolds numbers, buoyancy does not have time to lift this bubble far, during the very short impact duration. This bubble is also observed in liquid-onliquid impacts. ${ }^{18}$

The formation of the bubble is most clearly explained by the mandatory stagnation point in the air directly under the center of the incoming drop. The air flow under the drop will certainly resemble that of a stagnation point flow, i.e., the driving pressure will be radial from some center. The air at this center will not know which way to go until the drop fluid is right upon it, at which time the escape routes may have been blocked, by the contacting outer ring of fluid.

The size of the bubble can be roughly estimated from the photographs. Their diameter is of the order of $50 \mu \mathrm{m}$. The area of the dark region mentioned above is about $20000 \mu \mathrm{m}^{2}$, which gives an estimate of the thickness of the air trapped under the drop as $3.5 \mu \mathrm{m}$. The thickness of the boundary layer in the air rushing along the surface from under the drop can be estimated as $\delta=\sqrt{\nu T}=\sqrt{\nu L / U}$, where $L$ is the radius of the dark region and the $U$ the impact velocity. This gives an estimate of $\delta$ as $16 \mu \mathrm{m}$, which is of similar order considering the crudeness of the argument.

\section{E. Capillary waves during the contraction}

Following the maximum spreading of the drop, surface tension pulls the edge back, as demonstrated in Fig. 9. During this contraction phase, capillary waves on the lamellar surface become quite pronounced as surface tension becomes dominant and inertia and viscous forces disappear. This is shown in Fig. 28.

At the end of the expansion the edge of the lamella has become considerably thicker than the rest of the sheet. This is the stage where surface tension breaks up the front into disparate fingers or drops. Depending on the wettability of the surface these fingers are either pulled back or roll along the surface, forming the familiar fingers of a blot on a sheet of paper. ${ }^{7}$ This is also shown clearly in the drawings of Worthington for the mercury drop.

Capillary waves propagate from this stalled front backward toward the center of the drop. These waves originate all around the drop, but seem to eminate from the most intense disturbances, thus showing crests curved away from the drop center. This leads to interactions and radial interference patterns, visible as fuzzy radial lines. These capillary waves are entering a thin layer of fluid of variable depth. The depth is probably shallower toward the center, as is indicated by the fact that dewetting, when observed, usually takes place close to the drop center. The wavelength of the waves appears to decrease toward the center. This is also consistent with the dispersion relation for capillary waves, with the shortest waves traveling fastest. The wavelength also grows in time as the layer deepens.

\section{DISCUSSION AND CONCLUDING REMARKS}

\section{A. Mechanism of frontal evolution}

We have demonstrated how the fingering pattern of an impacting drop evolves during the expansion of the spreading lamella. The measured azimuthal phase speeds suggest strongly that surface tension is driving the evolution of the frontal shapes.

The strength of surface tension compared to viscosity and inertia can give an indication of to what extent capillary forces can dominate this evolution. 


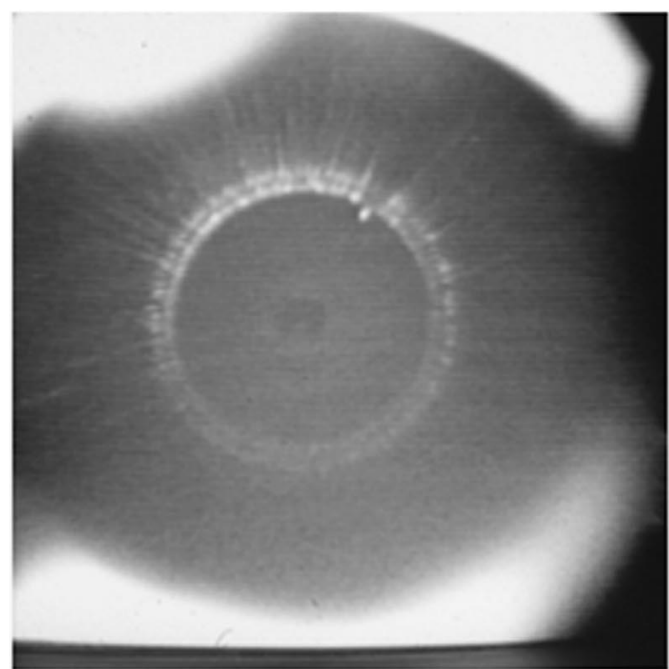

(a)

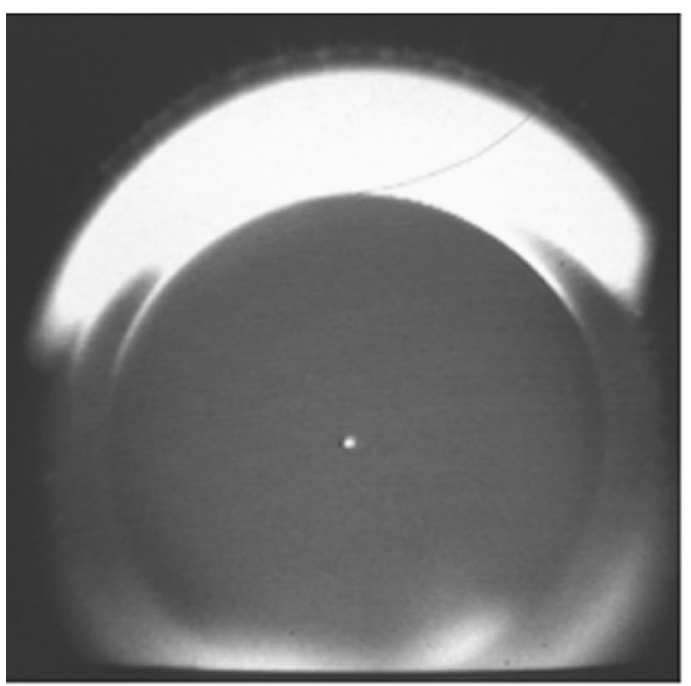

(c)

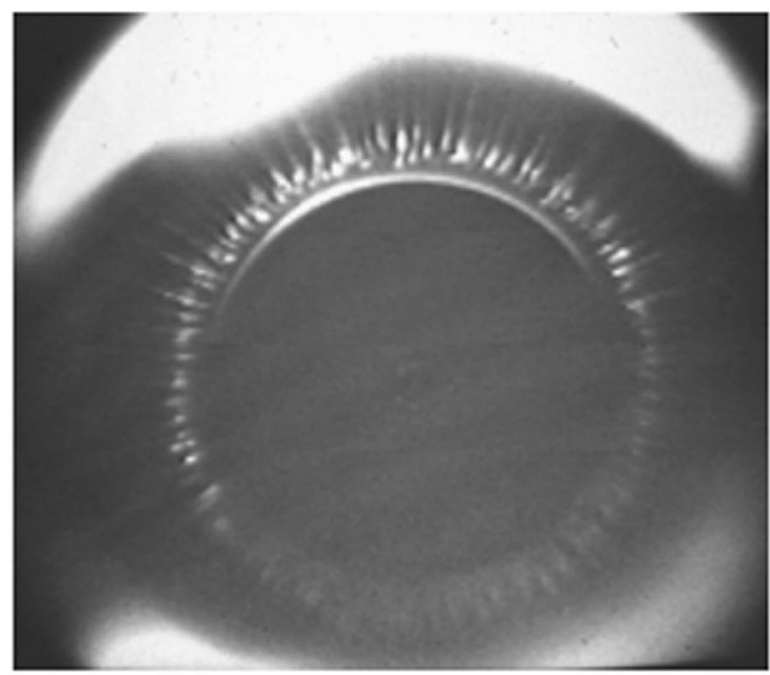

(b)

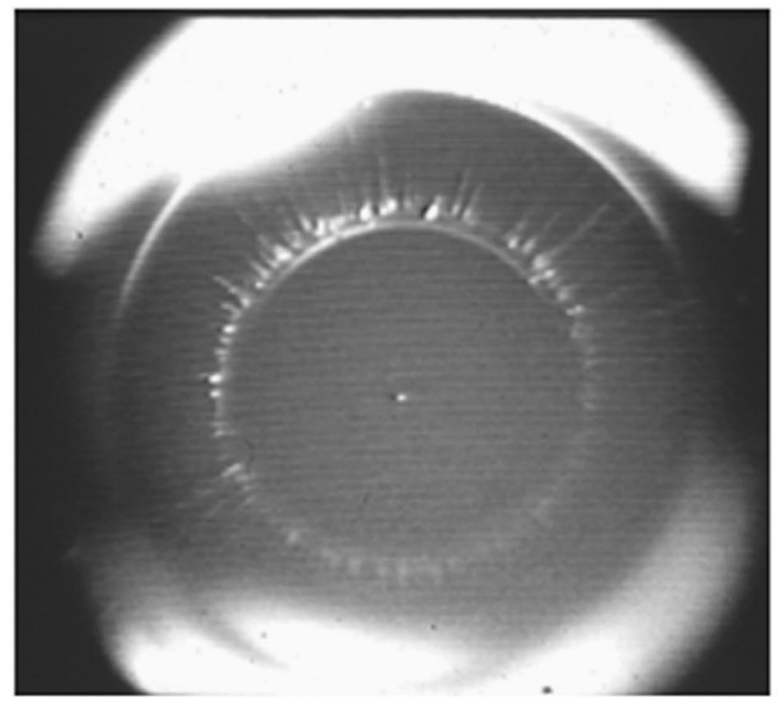

(d)

FIG. 26. The presence of azimuthal undulations, visible immediately after first contact. The formation of a small bubble under the center of the drop is also shown. Panel (d) has been double flashed, showing that the bubble has formed when the crease between the jet and the drop is about two-thirds of the drop diameter. Here $H=60 \mathrm{~cm}, D=5.5 \mathrm{~mm}$. The contacting regions span (a) $28 \%$, (b) $44 \%$, (c) $68 \%$, and (d) $39 \%$ of the drop diameter.

The relative strength of viscous and surface tension forces are usually estimated by the Capillary number, which at impact is $\mathrm{Ca}=\mu U / \gamma=0.6$. This, however, implies that the two forces are characterized by the same length scale, which is not correct for the case at hand.

These forces can be better estimated with the following assumptions about the shape of the front. Figure 29 shows a definition sketch of the advancing front. Here we use $z$ to denote the vertical coordinate and $\theta$ indicates the azimuthal angle. The viscous force decelerates the radial motion of the jet. Here we estimate its action at the lower boundary of a thin vertically oriented radial slice of the lamella, extending radially inward from the front by the length of a typical disturbance, approximately $\lambda$, as

$$
F_{\mathrm{visc}}=\mu \frac{\partial u_{r}}{\partial z} r d \theta \lambda \equiv \mu \frac{U}{h} r d \theta \lambda
$$

where $h$ is the local thickness of the lamella and $u_{r}$ is a disturbance to the spreading velocity $U$.

We next consider the surface tension acting on the same slice in the azimuthal direction. The strength of the surface tension relies on estimating the curvature of the free surface, between the fingers. With the current experimental setup we have no way of determining accurately the exact depth of the sheet, except we can determine from angled side views that the fingers are a continuation of a somewhat deeper ridge of fluid, as is implicit in the following argument. Here we assume the proportions shown in Fig. 29, which leads to the following estimate:

$$
F_{\text {surf }}=\gamma \frac{1}{r^{2}} \frac{\partial^{2} h(\theta)}{\partial^{2} \theta} r d \theta \lambda \approx \gamma \frac{h}{\lambda^{2}} r d \theta \lambda
$$

where $\gamma$ is again the surface tension coefficient. 


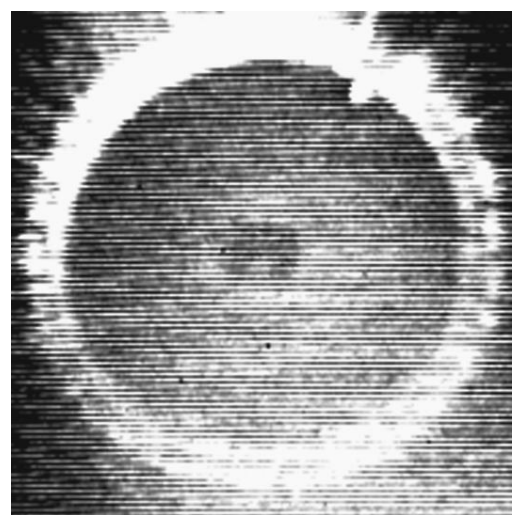

FIG. 27. Enhanced view of the dark region in Fig. 26(a).

Keeping in mind that the components of the two forces we have considered above do not act in the same direction, i.e., the azimuthal component of surface tension and the radial component of viscosity; their relative strength only indicates whether they can plausibly interact.

The ratio of viscosity to surface tension based on the above arguments has the form
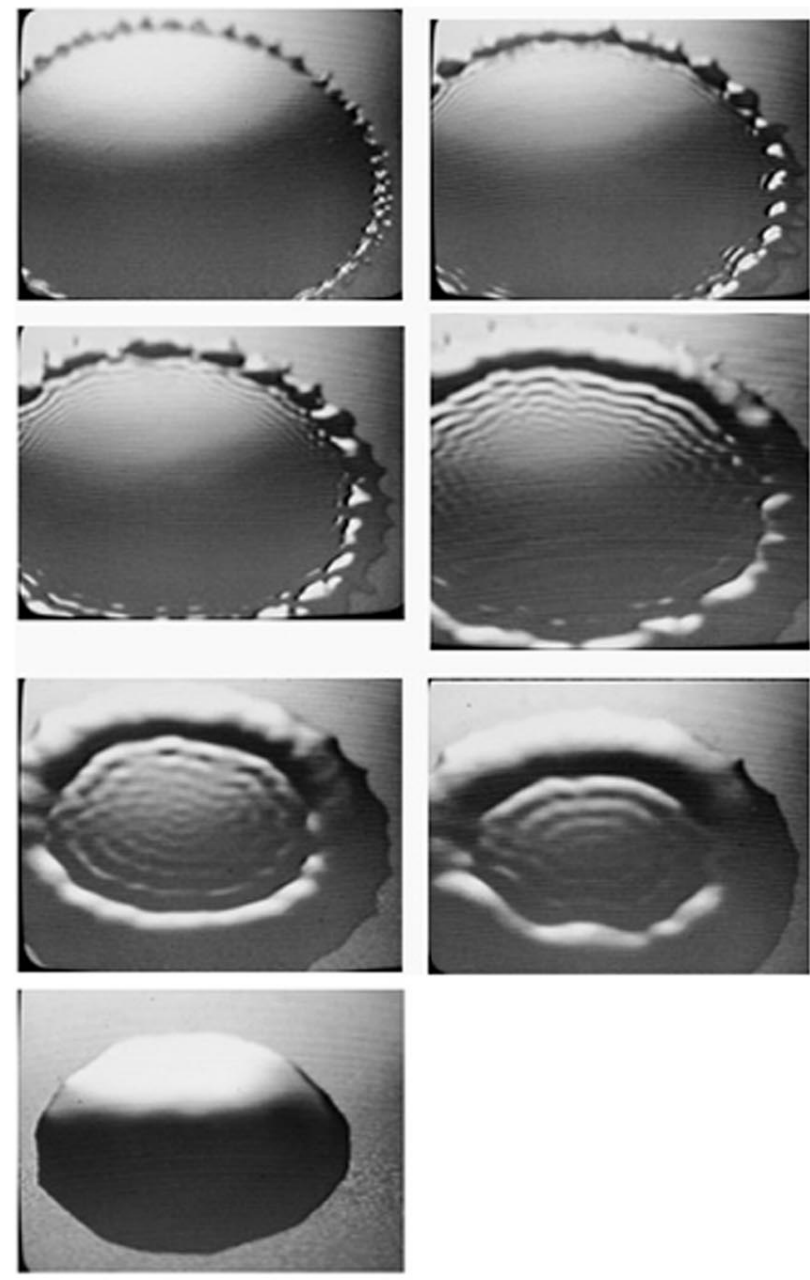

FIG. 28. The capillary waves on the surface of the drop during the recoil. The surface is anodized aluminum. The maximum spreading of the drop is approximately $2 \mathrm{~cm}$.
Side View
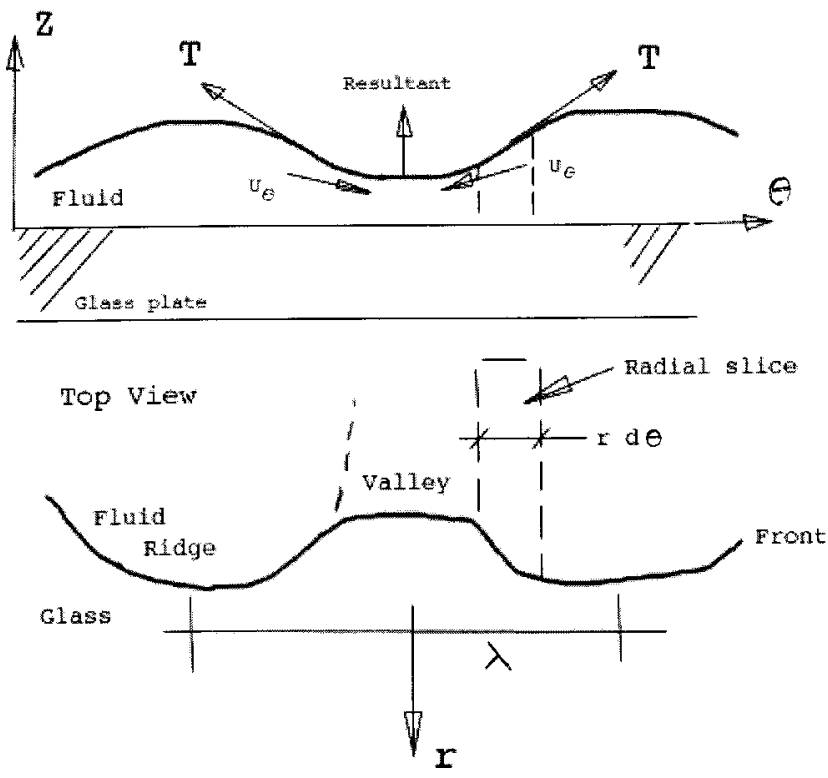

FIG. 29. A setup sketch showing the undulations due to the fingers.

$$
\frac{F_{\text {visc }}}{F_{\text {surf }}}=\frac{\mu U}{\gamma} \frac{\lambda^{2}}{h^{2}}
$$

by assuming $\lambda / h \approx 2.5$ and $U \approx 1 \mathrm{~m} / \mathrm{s}$; this gives a value of about 1 .

This should be considered in conjunction with the rapid deceleration of the jet and the rather slow azimuthal motions of the capillary waves. The interactions of the two forces could therefore affect the frontal shapes. In what follows we suggest just such a mechanism.

The pronounced acceleration of the fluid between merging fingers (shown in Figs. 18-21) could be explained by the following argument; see Fig. 29. The valley in the surface height between the adjacent fingers is acted on by the surface tension, which resultant attempts to pull up this surface. This pulls in fluid, by continuity, from the adjacent teeth, mostly from the fast moving fluid at the top of the layer. If there is an overshoot in the surface height, one could locally have a deeper fluid where there used to be a valley. This deeper liquid will be decelerated less, by viscosity, than the surrounding shallower layer, thus appearing to jet ahead. One could also wonder whether the fluid layer could leave the surface as it jets ahead, thus feeling negligible viscous deceleration. This is not likely, as no bubbles are observed to be entrained under the sheet.

In the above argument in Eq. (2) we have approximated the disturbance velocity $u_{r}$ with the spreading velocity $U$. The accuracy of this assumption is very dependent on the value of $\beta$, or, in other words, the $c / V$ ratio in Fig. 23(b). The realizations shown in Figs. 18 and 20 demonstrate that during merging the radial velocity at the valleys between the fingers can be as much as 50\% larger than that of the finger tips, justifying this scaling. 
Experiments using different surfaces with varying wetting properties and liquids having different surface tension coefficients, should alter this mechanism in testable ways.

\section{B. Number of fingers versus radial location}

The fundamental wavelength does not change significantly during the spreading. It may decline slightly, but the spread in the data is too large for a conclusive trend. In a recent review article, Rein $^{4}$ includes a photograph from the thesis of Loehr, ${ }^{6}$ showing that the number of prominent lamellar ridges remains constant during the expansion. The superposition of the numerous flashes used there may cloud the interpretation of that picture. Alternatively, the ridges may not follow the frontal evolution far upstream along the lamella. This photograph (which does not include specifics of the experimental conditions) may also be only of the initial evolution before the surface tension has altered the frontal shape significantly.

Direct comparison with the number of fingers observed by Marmanis and Thoroddsen ${ }^{7}$ is difficult, as the wettability of the surfaces used are completely different. They used a rough paper surface to study the fingering pattern left after the impact. The random orientation of the fibers in the paper probably neutralizes the contact angle effects. The final pattern left on the paper after the impact will also not leave signs of the pattern evolution during the expansion. One can argue that the large-amplitude fingers shown in Fig. 20 would lead to the dyed pattern on the paper. The number of fingers observed here $(H=50 \mathrm{~cm})$ at the end of the spreading is about 55, which is significantly larger than the value of about 42 obtained in Ref. 7 for a somewhat different value of the surface tension. The vast difference in wettability is the most likely cause of this difference.

\section{Possible surfactant effects}

The addition of the Fluorescein to the drop liquid does change the static surface tension significantly, as stated earlier. The Fluorescein molecule has indeed a structure somewhat similar to other known surfactants. The presence of surfactants may, however, not only change the value of the surface tension coefficient, but can also lead to Marangoni stresses. These stresses arise when surface tension varies along a liquid interface. Such spatial variations arise most commonly due to temperature variations along the surface, but can also arise, in the presence of surfactants, when the fluid surface is strained rapidly. The rapid dilatation of the surface reduces locally the surface concentration of the surfactant molecules, thereby increasing the local surface tension coefficient. The bulk surfactant concentration in the liquid will determine how quickly the surfactant molecules can be replenished. Therefore small concentrations of the surfactant molecules may cause greater Marangoni effects than larger concentrations. For our very high concentration of Fluorescein we expect rapid replenishing of the surfactant molecules at the surface, thus minimizing the effects of the surface dilatation on the dynamic surface tension.

Our imaging technique demands adding Fluorescein to the drop fluid. It therefore has to be considered as an integral part of the experimental conditions. How much our results will deviate from results obtained using pure water is unclear. The main phenomena of splitting and merging of fingers were indeed observed with pure distilled water. The impacts of distilled water drops could, however, only be studied using two flashes and with the camera looking at a small angular sector of the edge.

The Zhang and Basaran ${ }^{13}$ results show that surfactants affect the contraction and rebounding more strongly than the spreading phase of the impact. Pasandideh-Fard et al. ${ }^{12}$ found very little effects of surfactants, obtaining best results in their numerical simulations by ignoring those effects.

\section{Nature of the instability}

The nature of the fingering instability can be clarified using this data. The front evolves as it spreads, but as the fundamental wavelength does not change significantly, the instability proposed by Allen $^{8}$ is probably not the principal driving mechanism. We suggest an alternative, but similar, instability mechanism, i.e., a Rayleigh-Taylor instability of the fluid ring that first feels the presence of the solid surface and decelerates in the air right before hitting the plate. This is supported by the appearance of azimuthal undulations at first contact. The time duration of the deceleration is not easily predictable. The edge can also not be identified clearly from the earliest images. We have tried to count the number of radial intensity features around the outer edge of the contacting ring in the image in Fig. 26(b). The value of 85 is quite subjective, but is of the same order of magnitude as the final number of fingers, which for $H=60 \mathrm{~cm}$ is about 60 . The larger number is also qualitatively consistent with the slight downward trend in Fig. 14. The contact is at this stage about $2 \mathrm{~mm}$ wide, so the corresponding wave number $k$ is about $4 \times 10^{4} \mathrm{rad} / \mathrm{m}$. This number can be compared to the fastest growing wave number of the Rayleigh-Taylor instability. The growth rate of this instability including the stabilizing effects of surface tension takes the form ${ }^{20}$

$$
s^{2}(k)=g^{*} k\left(\frac{\rho_{2}-\rho_{1}}{\rho_{2}+\rho_{1}}-\frac{k^{2} \gamma}{g^{*}\left(\rho_{2}+\rho_{1}\right)}\right),
$$

where $s(k)$ is the growth rate of wave number $k$ and $g^{*}$ is here the net acceleration felt by the interface. Here the density of air $\rho_{1}$ is so much smaller than that of water $\rho_{2}$ that it can be ignored. Furthermore, the deceleration of the drop as it impacts the surface is so large that gravity can be ignored in the effective acceleration. Incorporating these assumptions into the above equation, the fastest growing wave number $k^{*}$ becomes

$$
k^{*}=\sqrt{\frac{g^{*} \rho_{2}}{3 \gamma}} .
$$

The deceleration of the drop takes place in a very short distance $\Delta$ and the rate of deceleration is $g^{*}=U^{2} / \Delta$. The value of $\Delta$ is difficult to estimate, but we can solve the above equation for the value needed to produce a wave number equal to that observed. This gives $\Delta=48 \mu \mathrm{m}$ and corresponds to a huge $25000 \mathrm{~g}$ of deceleration. The earliest contact of the ring [see Fig. 26(a)] appears to have a diameter of 
about $1 \mathrm{~mm}$, which spans about $10^{\circ}$ angle from the drop center. A plane cut through this angle will slice of a sliver of $25 \mu \mathrm{m}$ thickness, from the edge. The estimates of the boundary layer thicknesses in the air (see Sec. IV D) give a thickness of $32 \mu \mathrm{m}$ when one includes the boundary layers both on the drop and the solid surface.

The crudeness of these quantitative estimates can hardly be considered as conclusive proof for this mechanism, but these numbers do not seem to rule it out. The above instability is furthermore inviscid, whereas viscosity is known to affect the number of fingers. ${ }^{6,7}$ More detailed experiments or analyses, including viscosity and stabilizing azimuthal strain, are needed.

The undulations produced by this instability will be imprinted on the accelerating jet and subsequently be acted on by the proposed capillary-viscous interactions during the spreading.

\section{SUMMARY}

We have developed a new visualization technique to observe the evolution of the fingering instability of an impacting drop. The images show that the instability begins immediately at the first contact of the drop with the solid surface. We propose that the fundamental instability is a surface tension modified Rayleigh-Taylor instability of the rapidly decelerating annular ring of fluid that first touches the surface. These initial undulations are subsequently imprinted on the radially expanding jet. This generation schenario is significantly different from that proposed by Allen, ${ }^{8}$ i.e., a Rayleigh-Taylor at the viscously decelerating jet.

The number of fundamental disturbances, or fingers, remains approximately constant during the expansion. The frontal shapes do, however, evolve strongly during the spreading. This is characterized by widening and splitting of the fingers and subsequent merger of adjacent fingers, thus converting valleys into fingertips.

Capillary waves account reasonably well for the phase velocity of these disturbances.

\section{ACKNOWLEDGMENTS}

J.S. was supported by a JSPS Research Fellowship for Young Scientists No. 4941. We thank Ronald Adrian,
Haralambos Marmanis, Daniel Riahi, William Phillips, and James Phillips for their assistance and useful discussions.

${ }^{1}$ M. Rein, "Phenomena of liquid drop impact on solid and liquid surfaces," Fluid Dyn. Res. 12, 61 (1993).

${ }^{2} \mathrm{~A}$. M. Worthington, "On the forms assumed by drops of liquids falling vertically on a horizontal plate," Proc. R. Soc. London 25, 261 (1876).

${ }^{3}$ A. M. Worthington, "The splash of a drop," The Romance of Science Series (Richard Clay \& Sons, London, 1907); also A Study of Splashes (Longmans, Green, London, 1908).

${ }^{4}$ M. Rein, "Wave phenomena during droplet impact," IUTAM Symposium on Waves in Liquid/Gas and Liquid/Vapour Systems, edited by X. Morioka and X. Wijngaarden (Kluwer, Dordrecht, 1995), p. 171.

${ }^{5}$ K. F. Loehr and A. Lasek, "Splashing of drops," Arch. Mech. Stos. 42, 507 (1990).

${ }^{6}$ K. F. Loehr, "Etalement et eclatement de gouttes," Diplom De Doctorat de l'Universite Pierre et Marie Curie, Paris 6, 1990.

${ }^{7} \mathrm{H}$. Marmanis and S. T. Thoroddsen, "Scaling of the fingering pattern of an impacting drop," Phys. Fluids 8, 1344 (1996).

${ }^{8}$ R. F. Allen, "The role of surface tension in splashing," J. Colloid Interface Sci. 51, 350 (1975)

${ }^{9}$ C. D. Stow and M. G. Hadfield, “An experimental investigation of fluid flow resulting from the impact of a water drop with an unyielding dry surface,', Proc. R. Soc. London, Ser. A 373, 419 (1981).

${ }^{10}$ C. H. R. Mundo, M. Sommerfeld, and C. Tropea, "Droplet-wall collisions: Experimental studies of the deformation and breakup process," Int. J. Multiphase Flow 21, 151 (1995).

${ }^{11} \mathrm{~S}$. Chandra and C. T. Avedisian, "On the collision of a droplet with a solid surface,” Proc. R. Soc. London, Ser. A 432, 13 (1991).

${ }^{12}$ M. Pasandideh-Fard, Y. M. Qiao, S. Chandra, and J. Mostaghimi, "Capillary effects during droplet impact on a solid surface," Phys. Fluids 8, 650 (1996).

${ }^{13} \mathrm{X}$. Zhang and O. A. Basaran, "Dynamic surface tension effects in impact of a drop with a solid surface," J. Colloid Interface Sci. 187, 166 (1997).

${ }^{14}$ F. H. Harlow and J. P. Shannon, "The splash of a liquid drop,'” J. Appl. Phys. 38, 3855 (1967).

${ }^{15}$ J. Fukai, Z. Zhao, D. Poulikakos, C. M. Megaridis, and O. Miyatake, "Modeling of the deformation of a liquid droplet impinging upon a flat surface,' Phys. Fluids A 5, 2588 (1993).

${ }^{16}$ J. Fukai, O. Miyatake, Y. Shiiba, T. Yamamoto, D. Poulikakos, C. M. Megaridis, and Z. Zhao, "Wetting effects on the spreading of a liquid droplet colliding with a flat surface: Experiment and modeling," Phys. Fluids 7, 236 (1995).

${ }^{17}$ A. L. Yarin and D. A. Weiss, "Impact of drops on solid surfaces: selfsimilar capillary waves, and splashing as a new type of kinematic discontinuity," J. Fluid Mech. 283, 141 (1995).

${ }^{18}$ B. Peck and L. Sigurdson, "The three-dimensional vortex structure of an impacting water drop," Phys. Fluids 6, 564 (1994).

${ }^{19}$ J. Lighthill, Waves in Fluids (Cambridge University Press, Cambridge, 1978), p. 226.

${ }^{20} \mathrm{~S}$. Chandrasekhar, Hydrodynamic and Hydromagnetic Stability (Oxford University Press, Oxford, 1961), p. 435. 